\title{
Search Optimization, Funnel Topography, and Dynamical Criticality on the String Landscape
}

\author{
Justin Khoury and Onkar Parrikar \\ Center for Particle Cosmology, Department of Physics and Astronomy, University of Pennsylvania, \\ 209 South 33rd St, Philadelphia, PA 19104
}

\begin{abstract}
A striking feature of our universe is its near criticality. The cosmological constant and weak hierarchy problems, as well as the metastability of the electroweak vacuum, can all be understood as problems of criticality. This suggests a statistical physics approach, based on the landscape of string theory. In this paper we present a dynamical selection mechanism for hospitable vacua based on search optimization. Instead of focusing on late-time, stationary probability distributions for the different vacua, we are interested in the approach to equilibrium. This is particularly relevant if cosmological evolution on the multiverse has occurred for a finite time much shorter than the exponentially-long mixing time for the landscape. We argue this imposes a strong selection pressure among hospitable vacua, favoring those that lie in regions where the search algorithm is efficient. Specifically, we show that the mean first passage time is minimized for hospitable vacua lying at the bottom of funnel-like regions, akin to the smooth folding funnels of naturallyoccurring proteins and the convex loss functions of well-trained deep neural networks. The optimality criterion is time-reparametrization invariant and defined by two competing requirements: search efficiency, which requires minimizing the mean first passage time, and sweeping exploration, which requires that random walks are recurrent. Optimal landscape regions reach a compromise by lying at the critical boundary between recurrence and transience, thereby achieving dynamical criticality. Remarkably, this implies that the optimal lifetime of vacua coincides with the de Sitter Page time, $\tau_{\text {crit }} \sim M_{\mathrm{Pl}}^{2} / H^{3}$. Our mechanism makes concrete phenomenological predictions: 1 . The expected lifetime of our universe is $10^{130}$ years, which is $\gtrsim 2 \sigma$ from the Standard Model metastability estimate; 2 . The supersymmetry breaking scale should be high, $\gtrsim 10^{10} \mathrm{GeV}$. The present framework suggests a correspondence between the near-criticality of our universe and nonequilibrium critical phenomena on the landscape.
\end{abstract}

\section{Introduction}

Naturalness and symmetry have been the guiding principles of particle physics for the last fifty years. They were central in the development of the Standard Model (SM) and have steered Beyond-the-SM (BSM) model building. It appears increasingly doubtful, however, that these cherished principles can explain the finetunings currently facing theoretical physics. The lack of evidence for new physics at the Large Hadron Collider (LHC) suggests that Nature may have chosen the most parsimonious UV completion for the SM - a weakly coupled scalar field, with nothing else to stabilize the weak scale. The failure of direct detection experiments to discover Weakly Interacting Massive Particle (WIMP) dark matter, a natural by-product of low-scale supersymmetry (SUSY), only strengthens the case against new weak-scale physics. Last but not least is the cosmological constant, which remains steadfastly immune to any compelling natural explanation [1].

Perhaps we have been unlucky. New particles might lie just beyond the reach of the LHC, and WIMPs may be buried beneath the neutrino floor. But the current state of affairs has led some to ponder whether particle physics has entered a post-naturalness era [2].

A disturbing consequence of a grand "desert" above the weak scale is the metastability of the electroweak 
vacuum [3 17]. Within the SM the Higgs quartic coupling $\lambda(\mu)$ becomes negative at high energy, implying that our vacuum is metastable. The estimated lifetime of our vacuum is $\left[17^{1}\right.$

$$
\tau=10^{526_{-202}^{+409}} \text { years. }
$$

While much longer than the age of the universe, it is an uncomfortably close call. The quoted lifetime hinges on a delicate cancellation between an exponentially small number - the bounce factor, $\exp \left(-8 \pi^{2} / 3\left|\lambda\left(\mu_{\star}\right)\right|\right)$, which is exquisitely sensitive to $\lambda$ at the scale $\mu_{\star} \simeq 3 \times 10^{17} \mathrm{GeV}$ where it achieves a minimum; and an exponentially large number - the space-time volume of our universe 2 What makes the Higgs metastability particularly intriguing is that it relates the cosmological constant, which sets the characteristic 4-volume, and the weak scale, which sets the Higgs and top quark masses.

We believe this numerical conspiracy is no accident. It begs the question - why is our universe so precariously close to the edge of instability? Anthropic reasoning, which has been successfully applied to the vacuum energy [20] and the Higgs expectation value [21], does not seemingly offer any guidance, for it is difficult to conceive a priori how vacuum metastability is a pre-requisite for the existence of observers. (See [8, 22, 38] for cosmological implications of the Higgs metastability.)

The metastability of our vacuum can be interpreted as a problem of near-criticality [11] [3 Remarkably, other fine-tuning problems can also be viewed through the prism of criticality. Consider the weak hierarchy problem. As remarked in [40], if the Higgs mass spans the natural range $-M_{\mathrm{Pl}}^{4} \lesssim m_{h}^{2} \lesssim M_{\mathrm{Pl}}^{4}$, then the puzzle of the measured $\left|m_{h}^{2}\right| \ll M_{\mathrm{Pl}}^{2}$ is that it is so close to the critical point between an unbroken phase with $m_{h}^{2} \sim M^{2}$ and $\langle h\rangle \simeq 0$, and a badly broken phase with $m_{h}^{2} \sim-M^{2}$ and $\langle h\rangle \sim M_{\mathrm{Pl}}{ }^{4}$

Less well-defined but equally intuitive is the cosmological constant, whose minute value implies a nearlyflat universe. In some sense, Minkowski space represents a quantum critical point between de Sitter (dS) and anti-de Sitter (AdS) space-times, which have different asymptotics and dynamical stability properties 43, 44. Last but not least is the near scale-invariance of primordial density perturbations, whose spectral index is suggestive of a critical exponent. The mechanism traditionally invoked to generate these perturbations, namely slow-roll inflation, itself represents a near-critical phenomenon, with the inflaton interpolating between a nearly de Sitter phase (approximate conformal fixed point) and standard decelerating expansion.

\section{$1.1 \quad$ Landscape approach}

The near criticality of our universe strongly suggests a statistical physics approach, based on the idea that the parameters of the SM vary across a vast landscape of vacua, with the observed values determined environmentally. This is motivated by the exponentially large number of metastable states in string theory [45 48], together with the mechanism of eternal inflation [49 51 , to dynamically populate these vacua ${ }^{5}$

A significant challenge to this approach is the measure problem [55]. Since bubbles of all type are generated infinitely-many times in an eternally inflating universe, a regularization prescription (i.e., a measure) is required to count bubbles and define late-time probabilities. There are two broad classes of measures. The first class comprises global measures: given a global foliation specified by some global time coordinate $t$, one counts bubbles of different types on a late-time hypersurface $t=t_{\mathrm{c}}$, and then let $t_{\mathrm{c}} \rightarrow \infty$ 56 63. The

\footnotetext{
${ }^{1}$ To be clear, (1) is the characteristic time to form a bubble a true vacuum within our observable universe.

${ }^{2}$ The calculation of the tunneling rate per unit volume is done in flat space-time. Gravitational corrections are consistently small [18, 19.

${ }^{3}$ For instance, the decay rate per space-time volume is relatively close to the critical range for percolation [39.

${ }^{4}$ One should emphasize that even with $m_{h}^{2}>0$, the electroweak phase is spontaneously broken at the QCD scale by the Higgs coupling to the quark condensate. See 41, 42 for discussions of this other phase of the SM.

${ }^{5}$ Recently, the existence of metastable dS vacua in string theory has been questioned [52, 53, which has sparked a heated debate (e.g., see [54] and references therein). In this paper we will assume that the fundamental theory admits a rich landscape of vacua, including metastable dS vacua.
} 
resulting measure is independent of initial conditions, reflecting the attractor behavior of eternal inflation. A major drawback, however, is that it depends sensitively on the choice of global time variable [56 58 ].

The second class includes local measures, which focus on a space-time region around time-like geodesics. Examples include the past light-cone of world-lines (causal patch or causal diamond measure 64, 65]), a region bounded by the apparent horizon [66], or a space-like region around a world-line ("fat geodesic" [67]). In this paper we will primarily follow the "watcher" measure, defined by an ensemble of time-like geodesics or "watchers" 62, 63, 68, 69. For instance, the ensemble can be generated as follows [69]: starting with an observer in some initial dS vacuum, we follow all future "decohered" classical histories, thus generating an ensemble of world-lines weighted unambiguously by their quantum-mechanical branching ratios.

A feature common to all local measures is that a typical geodesic will eventually enter an AdS or Minkowski vacuum. An AdS region collapses within a Hubble time to a big crunch singularity, hence AdS vacua are usually assumed terminal. Minkowski vacua are also generally assumed to be terminal. Therefore, all but a measure zero of watchers will sample a finite number of bubbles. This regulates the infinities of eternal inflation 64, but introduces a dependence on initial conditions. In this work AdS and Minkowski vacua will be treated as terminal, and we will argue in Sec. 2.5 that under certain assumptions our results are largely independent of initial conditions.

Underlying these approaches to the landscape is the assumption that the multiverse has existed for a sufficiently long time, such that vacuum statistics have nearly settled to a stationary/equilibrium distribution. To be precise, denote by $f_{i}(t)$ the probability that a watcher occupies vacuum $i$ at time $t$. (Equivalently, $f_{i}(t)$ is the fraction of comoving volume occupied by vacuum $i$.) Asymptotically, this tends to a stationary distribution $\vec{f}^{(0)}$, which lies entirely in the subspace of terminal vacua, up to an exponentially-decaying piece $\delta \vec{f}=\vec{s} e^{q t}$, which is dominated by the longest-lived dS vacuum, called the dominant vacuum. Since the latter is unlikely to be anthropically hospitable, the frequency of hospitable vacua is determined by the tunneling rate from the dominant vacuum into hospitable ones. The hope is that, according to this measure, a universe like ours is statistically likely among all possible anthropically hospitable vacua. In other words, our universe ought to be generic or typical according to the measure. This is the principle of mediocrity.

The assumption that the multiverse has nearly settled into a stationary state is non-trivial. As with many complex systems [70, in particular spin glasses [71, the landscape features many long-lived metastable vacua, resulting in frustration and aging dynamics. Consequently the mixing time for the landscape globally is exponentially long [72. In the language of computational complexity, the number of vacua $N$ scales exponentially with the effective dimensionality $D$ of the landscape, that is, $N \sim \mathrm{e}^{D}$, and the problem of finding a vacuum within a specified hospitable range of vacuum energy has been argued to be NP-hard [73]. See [74 for further implications.

\subsection{Search optimization and funnel topography}

Instead of focusing on equilibrium distributions, our primary interest in this paper lies in the approach to equilibrium. This is particularly relevant if, as observed by Denef et al. [75, the multiverse has only existed for a finite time much shorter than the exponentially-long mixing time for the landscape. Specifically we assume that all watchers in our ensemble hit terminal vacua on a time scale much shorter than the landscape mixing time ${ }^{6}$ The finite-time perspective changes the relevant question for the likelihood of our vacuum. Instead of asking, What type of hospitable vacua occurs most frequently at steady-state according to the equilibrium probability distribution?, we are interested in the question - What hospitable vacua have the right properties to be easily accessed in the evolution [75: $?^{7}$

\footnotetext{
${ }^{6}$ This effectively imposes an "end-of-time" geometric cutoff 76 at a time much shorter than the mixing time.

${ }^{7}$ Like [75], we remain agnostic as to whether hospitable vacua end up harboring observers or not. Observers will not play a central role in our discussion.
} 


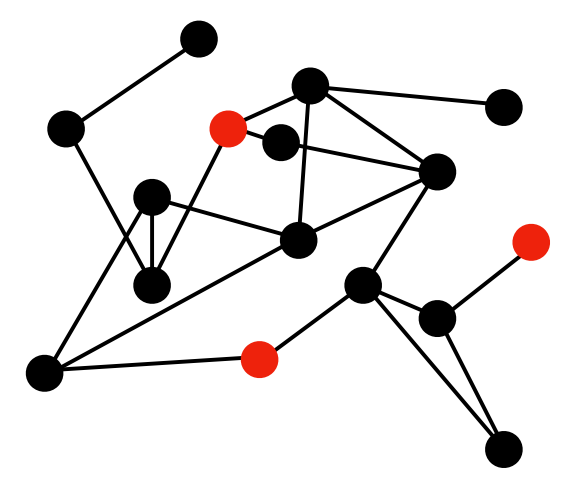

(a) Fiducial region
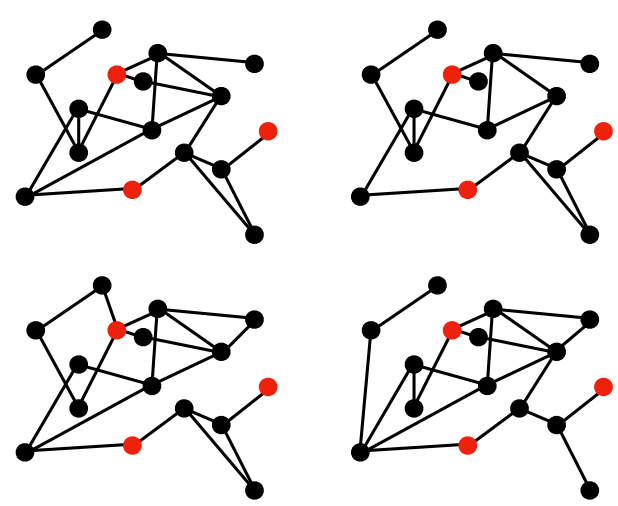

(b) Replicas with small variations

Figure 1: a) A fiducial region of the landscape, modeled as a weighted, undirected network. Nodes are metastable dS vacua, while links are the relevant transition rates. The red nodes are hospitable vacua. Tunneling rates into AdS/Minkowski vacua (not shown) introduce a rate of death. b) In the vastness of the landscape, we imagine there are many replicas of the fiducial region, each with slight variations. This is illustrated here with small changes in the network topology. More generally, we also expect variations in other physical parameters.

This translates to a search optimization problem: vacua that are easily accessed reside in optimal regions where the standard search algorithm is particularly efficient 8 A goal of this paper is to make precise the conditions for optimality and derive phenomenological implications for hospitable vacua lying in optimal regions of the landscape. As we will see, the optimality criteria will be independent of the choice of time variable, as well as whether vacua are weighted by comoving or physical volume. Furthermore, we will show that optimal regions are characterized by critical dynamics, thereby suggesting a connection between the near-criticality of our universe and non-equilibrium critical phenomena on the landscape.

To be clear, the approach does not claim to solve the measure problem. Ultimately the likelihood for our vacuum to reside in an optimal region $v s$ a sub-optimal one hinges on the choice of measure. We will try to carefully delineate which aspects of the calculation are sensitive to the measure, and which are not.

Consider a finite region of the landscape comprised of $N \gg 1 \mathrm{dS}$ vacua, large enough to include $N_{\text {target }} \ll$ $N$ hospitable vacua. ${ }^{9}$ We only explicitly keep track of dS vacua - the only role of AdS/Minkowski terminals is to introduce a "death" rate for watchers. In other words, the occupational probabilities $f_{i}(t)$ are conditional on the watcher not having transitioned yet to a terminal vacuum and died. Treating the region as a closed system for the moment, and ignoring bubble collisions, the $f_{i}(t)$ 's satisfy a linear Markov process 61 63, with transition rates governed by Coleman-De Luccia (CDL) instantons [78 80].

Thus the region can be modeled as a weighted and undirected network of $N$ nodes, with the set of links specifying the topology of the network (Fig. 1(a) [81. As his/her space-time neighborhood undergoes a sequence of transitions, the watcher is performing a random walk on this network (Sec. 2). The subject of random walks on graphs (or networks) is a venerable branch of mathematics, but in the natural sciences it has enjoyed an explosion of activity in recent years [82 84. This is in large part due to their relevance to a myriad applications, from biological networks, to the internet, to epidemic propagation.

In the vastness of the landscape, we imagine that our ensemble of watchers will sample an ensemble of landscape regions, comprised of many replicas of the fiducial region, each with slight variations (Fig. 1(b)).

\footnotetext{
${ }^{8}$ The link between optimization algorithms and the cosmological constant was also explored in [77.

${ }^{9}$ Although it is helpful to cast the discussion initially in terms 'hospitable' and 'inhospitable' vacua, our natural selection mechanism will lead to phenomenological predictions that rely as little as possible on anthropic reasoning.
} 


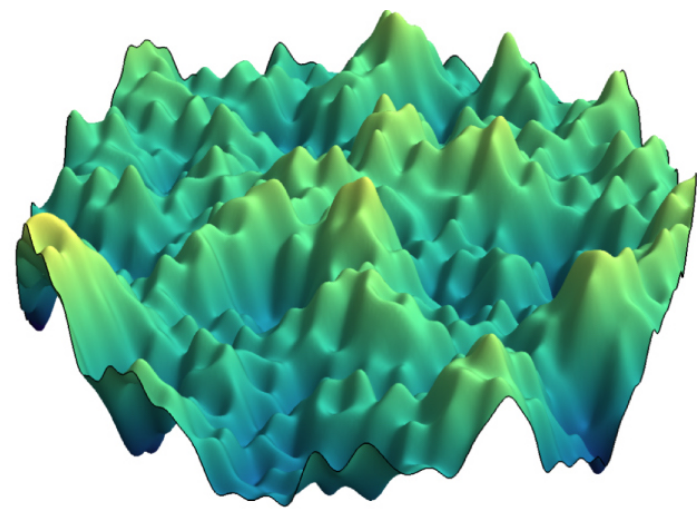

(a) Generic, frustrated region

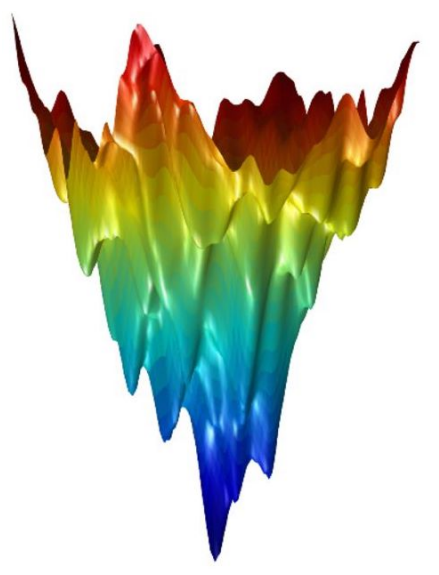

(b) Optimal, funnel-like region

Figure 2: a) A generic region in the landscape typically has multiple vacua whose only allowed transitions are upward jumps. Because upward jumps have exponentially suppressed transition rates, the dynamics are frustrated, resulting in exponentially long search times. (Credit: Chiara Cammarota.) b) An optimal region is characterized by all vacua having at least one allowed downward transition, except for the lowest-lying vacuum. Its landscape topography is that of a funnel, akin to the free energy landscape of proteins and the loss function of well-trained deep neural networks. (Credit: Reproduced from [94.)

For concreteness, we assume all regions have on average $N=\mathrm{e}^{D}$ vacua ${ }^{10}$ and the same underlying statistical distribution of potential energies, but otherwise differ slightly from each other in their network topology and transition rates. We will argue that within a generic representative region of the ensemble, the average time to access hospitable vacua likely scales exponentially with $D$ - consistent with the NP-hard complexity class of the general problem. However, assuming the ensemble is large enough, there will be exceptional regions where the search for hospitable vacua is optimal, characterized by polynomial search time.

Quantitatively, our figure of merit is a dimensionless mean first-passage time (MFPT) 85, a widelyused measure of random walk efficiency [86 88. The average MFPT, $\mathcal{T}_{\text {MFPT }}$, also known as Kemeny's constant [89, quantifies the average time taken by a random walker to reach a target vacuum, randomlyselected according to the stationary distribution. As reviewed in Sec. 3 the average MFPT admits a simple expression as a spectral sum over the eigenvalues of the transition matrix. The result is, however, most intuitively stated in the "downward" approximation [90, 91, which neglects the exponentially-small "upward" CDL transitions 92 :

$$
\mathcal{T}_{\mathrm{MFPT}} \simeq \sum_{i \neq \text { lowest }}^{N} \frac{1}{\kappa_{i} \Delta t} .
$$

Here, $\Delta t$ is a unit global time step, $\kappa_{i}$ is the total rate out of vacuum $i$, and the sum excludes the lowestlying vacuum which is necessarily stable in the downward approximation. Remarkably, 22 is identical to the mean transport time in quenched disordered media [93]. The result is intuitively clear - the MFPT is the sum over the characteristic number of waiting time steps, $\left(\kappa_{i} \Delta t\right)^{-1}$, for all metastable vacua in the region. Importantly, since $\kappa_{i}$ is the decay rate per unit time $t$, the dimensionless MFPT is manifestly timereparametrization invariant, i.e., independent of the choice of $t$. Furthermore, a well-known and remarkable property of Kemeny's constant is that it is independent of the initial node for the random walk.

Now, a generic region in the ensemble (sketched in Fig. 2(a) will typically include one or more metastable

\footnotetext{
${ }^{10}$ Recall that $D$ is the dimensionality of the landscape, controlled by the number of underlying moduli.
} 
vacua whose only possible transitions involve up-tunneling. In the strict downward approximation, these vacua are absolutely stable, but more precisely at sub-leading order their decay rate is exponentially suppressed. The average MFPT in such generic regions is exponentially long. A watcher is overwhelmingly likely to tunnel to an AdS (or Minkowski) terminal well before accessing a hospitable vacuum. If the ensemble is large enough, however, there ought to be rare but optimal regions, characterized by all vacua having at least one allowed downward transition to another $\mathrm{dS}$ vacuum (except of course for the lowest-lying vacuum). As sketched in Fig. 2(b), optimal regions have the topography of a broad valley or funnel. Furthermore, to minimize (2) downward transitions should be as fast as possible (though importantly, as we will see later, transition rates are bounded from above by other considerations). This results in power-law search time.

This is strikingly reminiscent of the protein folding problem [95, 96. Like the string landscape, the number of protein conformations (metastable vacua) scales exponentially with the number of amino acids (degrees of freedom). Correspondingly, the problem of finding the minimum energy state (so-called native state) is NP-complete [97. Physically, generic sequences of amino acids get trapped in long-lived metastable conformations, resulting in exponentially-long relaxation time. Yet naturally-occurring proteins fold efficiently to their native state. This is Levinthal's paradox [98. Nature's solution is of course evolution. The energy landscape of real proteins is characterized by high-energy unfolded states connected to the lowest-energy native state by a relatively smooth funnel. This is known as the principle of minimal frustration [95.

Deep learning offers a similar narrative. Finding the global minimum of the loss function of deep neural networks has long been known to be NP-complete 99. Recent improvements in visualization techniques have revealed a tantalizing connection between network architecture and loss function topography [100. Deep neural networks with poor training parameters (or overtrained networks) have a highly non-convex landscape, featuring many local minima, whereas well-trained networks that easily generalize correspond to smooth, funnel-like landscapes. Furthermore, it has been shown recently that the connectivity matrix of well-trained, state-of-the-art deep neural networks have power-law spectral density [101, which is welldescribed by heavy-tailed random matrix theory [102. This indicates that well-trained networks operate at criticality. The connection between search optimization and criticality will be a recurring theme in our discussion.

\subsection{Recurrence $v s$ transience}

We have so far approximated regions in the ensemble as closed systems, ignoring the exchange of probability with their surroundings. More realistically, regions are of course open systems, which allows the possibility that a random walker will escape a region before landing on a target vacuum. Optimal regions should therefore reach a comprise between efficient sampling, which tends to minimize the MFPT but increases the likelihood of escape, and oversampling, which minimizes the chance of escape but results in sub-optimal MFPT.

Treating regions as open systems would require modeling their environment, which in turn may introduce unwanted model-dependence in our analysis. Alternatively we propose to study a proxy requirement that relies solely on the intrinsic dynamics within a given region. Specifically, we consider the hypothetical limit of infinite volume, $N \rightarrow \infty$, and demand that random walks are recurrent in this limit. In recurrent walks, random walkers are certain to return to their starting point, and will do so infinitely-many times in the future. Equivalently, in recurrent walks every site will be visited with probability one. In contrast, for transient walks there is a finite probability that random walkers never return to their original site.

To be clear, at the end of the day our ensemble still consists of finite- $N$ regions. But the behavior of random walks in the $N \rightarrow \infty$ limit informs us on the potential impact of the environment of regions. Although not technically equivalent to modeling regions as open systems, we view the requirement of recurrence as a reliable proxy for efficient sampling of regions. Recurrent walks efficiently explore any region around their 
starting point, whereas transient walks tend to escape to infinity 11

In Sec. 4 we review the criterion for recurrence and show that it can be phrased in terms of a parameter $\mathcal{R}$, which is simply related to the average MFPT:

$$
\mathcal{R} \equiv \frac{\mathcal{T}_{\mathrm{MFPT}}}{N} .
$$

This can be interpreted as a mean residency time. Random walks are recurrent if $\mathcal{R}$ diverges as $N \rightarrow \infty$. In the downward approximation, this criterion reduces to

$$
\mathcal{R} \simeq\left\langle\frac{1}{\kappa_{i} \Delta t}\right\rangle \underset{N \rightarrow \infty}{\longrightarrow} \infty \quad \text { (recurrence) }
$$

where $\langle\ldots\rangle$ denotes the average over all vacua in the region. It should be intuitively clear that (4) is sensitive to the decay rate of the lowest-lying, and generically most stable, vacua. Random walks will be recurrent if the average decay rate diverges sufficiently fast as $V \rightarrow 0$.

\subsection{Dynamical criticality and natural selection}

We now come to a key result of this paper. Search optimization is defined by two competing requirements: search efficiency, which requires fast transition rates to minimize the average MFPT, and sweeping exploration, which requires sufficiently long-lived vacua to achieve recurrence. Optimal regions reach a compromise by having the shortest MFPT compatible with recurrence, i.e., $\mathcal{R}$ should diverge as $N \rightarrow \infty$ but at the slowest possible rate. In other words, optimal regions lie at the critical boundary between recurrence and transience.

We will show in Sec. 5 that optimality is achieved in regions characterized by vacua with average proper lifetime scaling as

$$
\tau_{\text {crit }}(V) \sim \frac{M_{\mathrm{Pl}}^{5}}{V^{3 / 2}} \sim \frac{M_{\mathrm{Pl}}^{2}}{H^{3}} \quad \text { as } V \rightarrow 0 .
$$

Remarkably, this optimal lifetime coincides with the dS Page time [104]. The dS Page time has appeared in other inflationary studies before [105-108. In slow-roll inflation, for instance, it marks the phase transition to eternal inflation [109, 110. The connection between the dS Page time and dynamically critical random walks on the landscape is surprising, and we do not yet have a compelling physical explanation for its occurrence.

The optimal lifetime (5) implies that $\mathcal{R}$ diverges as $\log N$, which signals dynamical criticality. A similar non-equilibrium phase transition occurs in quenched disordered media, when the probability distribution for waiting times reaches a critical power-law [93. The recurrence/transience boundary can be interpreted as critical from two points of view. Firstly, from a computational perspective, it represents a transition in the scaling of the recurrence measure $\mathcal{R}$ as a function of the effective dimensionality $D=\ln N$. Recurrence typically results in a power-law divergence in $N \sim \mathrm{e}^{D}$, and hence exponential in $D$. The critical case instead diverges as $\log N \sim D$, and therefore polynomially in $D$. More precisely, the large $N$ behavior of $\mathcal{R}$ delineates regions in the landscape into three basic types:

$$
\mathcal{R} \sim\left\{\begin{array}{ccc}
\operatorname{Exp}(D) & \cdots & \text { (recurrent but high complexity) } \\
\operatorname{Poly}(D) & \cdots & \text { (recurrent and low complexity) } \\
\text { Constant } & \cdots & \text { (low complexity but transient) }
\end{array}\right.
$$

where the second line corresponds to a critical boundary between the other two phases. The phase transition of interest is akin to computational phase transitions, which have the subject of much activity in recent

\footnotetext{
${ }^{11} \mathrm{~A}$ classic result in the theory of random walks is Pólya's theorem, which states that a simple random walk on a regular $d$-dimensional lattice is recurrent for $d \leq 2$, and transient for $d>2$ [103]. As mathematician Shizuo Kakutani famously quipped, "A drunken man will find his way home, but a drunken bird may get lost forever."
} 
years 111, 112. Secondly, from a dynamical perspective, it delineates regions of stability (recurrence) and instability (transience). Indeed, a pair of random walks starting from the same site will meet infinitely-many times in the recurrent case, but are certain to eventually never meet again in the transient case.

Thus the joint demands of sweeping exploration, defined by recurrence, and minimal oversampling, defined by minimal MFPT, select regions of the landscape that are tuned at criticality. As such our mechanism realizes a form of self-organized criticality [113-115]. It is consistent with the dynamical criticality hypothesis [116, which states that complex systems maximize their computational capabilities at the phase transition between stable and unstable dynamical behavior. This idea goes back to random boolean networks [117] and cellular automata [118, 119. Specifically, cellular automata with certain "edge of chaos" dynamical rules (Class IV per Wolfram's classification) are capable of universal computation, exhibiting long-lived and complex transient structures [120 124]. Recurrent neural networks [125, 126], specifically echo state networks [127, achieve maximal computational power for vanishing Lyapunov exponent [128. Moreover, as mentioned earlier, state-of-the-art deep neural networks are characterized by connectivity matrices with power-law spectral densities described by universality classes.

In biology, dynamical criticality is believed to be evolutionarily favorable. Brain activity operates in a critical state between stability, characterized by damped response to stimuli, and epileptic chaos, characterized by exponentially sensitive response [129 132]. Examples of dynamical criticality abound in the natural world [133, from gene expression [134, to cell growth [135], to flock dynamics [136, 137]. It has been conjectured that this was evolutionarily selected because dynamical criticality offers complex systems an ideal trade-off between robust, reproducible response and flexibility of adaptation to a changing environment.

Similarly our mechanism can be interpreted as natural selection of watchers, by analogy with optimal foraging theory [138, a rich subject in evolutionary ecology. A watcher in a given region can be thought of as a forager searching for scarce resources (hospitable vacua). The network topology and transition rates in that region (foraging site) define the foraging strategy of the watcher, while the probability of tunneling into terminal vacua encodes the risk of predation. Watchers with inefficient foraging strategies die before finding resources, while those with optimal foraging strategy find resources early on. This can be understood as natural selection of watchers, with 'fitness' defined as short average MFPT relative to the characteristic decay time into terminals. Furthermore, the optimal search strategy corresponds to critical dynamics ${ }^{12}$

\subsection{Phenomenological implications}

The search optimization mechanism makes concrete phenomenological predictions for vacua in optimal regions, described in Sec. 6. We should stress emphatically that these predictions do not rely on anthropic reasoning - they follow generally from optimality.

First and foremost, the critical transition rate (5) implies an optimal lifetime for our vacuum,

$$
\tau \sim \frac{M_{\mathrm{Pl}}^{2}}{H_{0}^{3}} \sim 10^{130} \text { years }
$$

This explains the metastability of the electroweak vacuum. Thus, given the observed vacuum energy $\sim H_{0}^{2}$, we predict the optimal lifetime for our vacuum, which in turn is sensitive to electroweak physics (in particular the Higgs and top quark masses). Quantitatively, the predicted lifetime agrees with (1) to within $\gtrsim 2 \sigma$. Closer agreement can be achieved if the top quark is slightly heavier, $m_{\mathrm{t}} \simeq 174.5 \mathrm{GeV}$, which can be viewed as a prediction. This assumes, of course, that the SM is valid all the way to the Planck scale. New physics at

\footnotetext{
${ }^{12}$ The idea that natural selection can play a role in cosmology was proposed long ago by Smolin [141 144]. His scenario postulates that space-time regions inside black holes form new universes. Whenever this happens, theory parameters undergo small random mutations which eventually favor universes that maximize black hole production. Another example of natural selection in the landscape was proposed in [145].
} 
intermediate scales, such as right-handed neutrinos with mass of $10^{13}-10^{14} \mathrm{GeV}$ [139], can bring the expected lifetime of our vacuum closer to the optimal prediction (7).

In general, our mechanism selects regions with efficient transition rates, particularly for low-lying vacua, and thus gives a raison d'être for the inferred Higgs metastability. New physics at scales below the SM instability scale, $\sim 10^{10} \mathrm{GeV}$, that affect the metastability of our vacuum, are disfavored by our mechanism. A prime example is low-scale SUSY [140]. As argued in Sec. 6, a natural prediction of our mechanism is that the SUSY-breaking scale is $\gtrsim 10^{10} \mathrm{GeV}$. Thus optimal regions of the landscape are characterized by very high-scale SUSY breaking, which is consistent with the absence of low-scale SUSY at the LHC.

\section{Random Walks on Complex Networks}

Consider a finite region of the landscape, comprised of $N \gg 1$ metastable dS vacua with potential energy $V_{j}>0$, where $j=1, \ldots, N$. We assume that $N$ is large enough that the region includes some number of hospitable target vacua. Statistically the $V_{j}$ 's are assumed to be drawn from some fixed probability distribution, which, at least for $V_{j}$ much smaller than the fundamental scale, we may assume to be a uniform distribution [146]. (More precisely, [146] argues for a uniform probability distribution in the narrow range of "anthropically allowed" vacua.) The region also contains AdS/Minkowski terminal vacua, which act as absorbing nodes or probability sinks ${ }^{13}$ We will not explicitly keep track of terminals - their only role is to introduce a rate of "death" for watchers. In other words, the occupational probabilities $f_{i}(t)$ defined below are conditional on a watcher not having yet transitioned to a terminal vacuum and died.

The region is modeled as a network (or graph) of $N$ sites/nodes representing the different vacua (Fig. 1(a)], with links denote the relevant transition rates. For the time being, the region is approximated as a closed system, ignoring the exchange of probability with its surroundings. We will relax this assumption in Sec. 4 Following the seminal papers by Garriga, Vilenkin and collaborators 61, 62, cosmological evolution on the landscape can be modeled as a simple Markov process. Let $f_{i}\left(\tau_{i}\right)$ denote the probability that the watcher occupies vacuum $i$, as a function of the local proper time $\tau_{i}$. Equivalently, $f_{i}$ is the fraction of total comoving volume occupied by vacuum $i$. The occupation probability satisfies a forward master equation 14

$$
\mathrm{d} f_{i}=\sum_{j} \mathrm{~d} \tau_{j} \kappa_{i j}^{\text {proper }} f_{j}-\mathrm{d} \tau_{i} \sum_{r} \kappa_{r i}^{\text {proper }} f_{i}
$$

where $\kappa_{i j}^{\text {proper }}$ is the $j \rightarrow i$ transition rate. We define the time variable $t$ as 62 , 63]:

$$
\mathrm{d} \tau_{i}=\mathcal{N}_{i} \mathrm{~d} t,
$$

where $\mathcal{N}_{i}$ is a lapse function. In a discrete setting, we can think of $t$ as a uniform discrete counter for transitions. In our analysis we will remain agnostic about the choice of time. Crucially later on we will focus on random walk statistics, specifically recurrence $v s$ transience, that are time-reparametrization invariant.

In terms of time $t$, the master equation (8) takes the form

$$
\frac{\mathrm{d} f_{i}}{\mathrm{~d} t}=\sum_{j} M_{i j} f_{j}
$$

where $M_{i j}$ is the transition matrix:

$$
M_{i j} \equiv \kappa_{i j}-\delta_{i j} \sum_{r} \kappa_{r j}
$$

\footnotetext{
${ }^{13}$ In a separate paper we will consider an alternative, albeit more speculative possibility, namely that collapsing AdS regions can sometimes bounce and avoid big crunch singularities, as considered by 68, 147.

${ }^{14}$ The master equation is linear in the transition rate because it ignores the effect of bubble collisions. More generally, bubble collisions can be encoded perturbatively as corrections that are non-linear in the transition rates [148].
} 
and

$$
\kappa_{i j}=\mathcal{N}_{j} \kappa_{i j}^{\text {proper }}
$$

is the $j \rightarrow i$ transition rate as per unit time $t$. Henceforth we will work with $t$ for convenience; we will change back to proper time at the end by using (12). By construction, the sum of any column of $M$ vanishes, $\sum_{i} M_{i j}=0$, which implies

$$
\sum_{i=1}^{N} f_{i}=1 .
$$

Thus probability is conserved, since by assumption the watcher has not yet died.

It is convenient to expand $f_{i}(t)$ in terms of Green's functions $P_{k i}(t)$ :

$$
f_{i}(t)=\sum_{j} P_{i j}(t) f_{j}(0) .
$$

Here, $P_{k i}(t)$ is the $N \times N$ occupational probability matrix that the system is in state $k$ at time $t$, starting from state $i$ at $t=0$. It satisfies 10 )

$$
\frac{\mathrm{d} P_{k i}}{\mathrm{~d} t}=\sum_{j} M_{k j} P_{j i},
$$

with initial condition $P_{k i}(0)=\delta_{k i}$. More succinctly, in matrix notation,

$$
\frac{\mathrm{d} P}{\mathrm{~d} t}=M P ; \quad P(0)=\mathbb{1} .
$$

The solution is

$$
P(t)=\mathrm{e}^{M t} .
$$

\subsection{Spectrum of $M$ and statistical equilibrium}

The transition matrix $M$ satisfies the following properties: $i$ ) its off-diagonal elements are positive semidefinite, $M_{i j}=\kappa_{i j} \geq 0$, for $i \neq j$; and $i i$ ) the sum of any column vanishes, $\sum_{i} M_{i j}=0$. We further assume that it is irreducible, i.e., there exists a sequence of transitions $i \rightarrow \ldots \rightarrow j$ connecting any $(i, j)$ pair.

With these assumptions, one can show by invoking Perron-Frobenius' theorem that the maximal eigenvalue of $M$ is non-degenerate and vanishes,

$$
\lambda_{1}=0,
$$

while all other eigenvalues are strictly negative, $0>\lambda_{2} \geq \ldots \geq \lambda_{N}\left[62\right.$. The zero-mode $v_{M}^{(1)}$ satisfies

$$
M v_{M}^{(1)}=0 .
$$

Per (10), this state sets the stationary distribution,

$$
f_{i}^{\infty} \equiv f_{i}(t \rightarrow \infty)=v_{M i}^{(1)} .
$$

The characteristic time required to reach this distribution, known as the mixing time, is set by the smallest (in magnitude) non-zero eigenvalue:

$$
t_{\text {mix }}=\left|\lambda_{2}\right|^{-1}
$$




\subsection{Transitions rates}

While transitions between any two vacua are possible quantum mechanically, whether or not they are connected by an instanton [149], in practice the decay rate out of a given vacuum $j$ is dominated by transitions to a small number of destination vacua. These are shown in Fig. 1(a) as links between nodes. The set of links specifies the topology of the network.

For concreteness we assume such transitions occur through Coleman-De Luccia (CDL) tunneling, in which case the $j \rightarrow i$ transition rate is of the weighted form

$$
\kappa_{i j}=\frac{A_{i j}}{w_{j}} ; \quad A_{i j}=A_{j i} .
$$

The adjacency matrix $A_{i j}$ is given by, in the saddle-point approximation,

$$
A_{i j}=\left(\Lambda^{4} \mathrm{e}^{-S_{\text {bounce }}}\right)_{i j},
$$

where $S_{\text {bounce }}$ is the bounce action, and $\Lambda^{4}$ is a characteristic scale set by the fluctuation determinant. The weight $w_{j}$ of each node is

$$
w_{j}=H_{j}^{3} \mathcal{N}_{j}^{-1} \mathrm{e}^{S_{j}} .
$$

The factor of $H_{j}^{3}$ arises from the volume of a Hubble patch of de Sitter false vacuum, where $H_{j}^{2}=V_{j} / 3 M_{\mathrm{Pl}}^{2}$. The lapse function converts from a rate per unit false-vacuum proper time to a rate per unit time $t$, via (12). The de Sitter entropy of the false vacuum, $S_{j} \equiv \frac{48 \pi^{2} M_{\mathrm{P}}^{4}}{V_{j}}$, appears in the exponential, hence low-lying vacua are exponentially weighted.

Since $A_{i j}$ is symmetric 92 , the rate satisfies a form of detailed balance,

$$
\frac{\kappa_{j i}}{\kappa_{i j}}=\frac{w_{j}}{w_{i}}=\frac{H_{j}^{3}}{H_{i}^{3}} \frac{\mathcal{N}_{i}}{\mathcal{N}_{j}} \mathrm{e}^{-\left(S_{i}-S_{j}\right)}
$$

That is, the forward vs backward rate is fixed by the relative weight of the nodes.

\section{$2.3 \quad$ Weighted networks}

The simple weighted form 222 allows for a number of analytical results 150. To begin with, despite not being symmetric, $M$ has real eigenvalues, and its eigenvectors form a complete basis. To see this, note that in matrix notation

$$
M=Z W^{-1},
$$

where

$$
Z_{i j} \equiv A_{i j}-\delta_{i j} \sum_{r} A_{r j} ; \quad W=\operatorname{diag}\left(w_{1}, w_{2}, \ldots, w_{N}\right) .
$$

Then, define the auxiliary matrix

$$
\Sigma \equiv W^{-1 / 2} Z W^{-1 / 2}=W^{-1 / 2} M W^{1 / 2} .
$$

This matrix is symmetric, hence its eigenvalues are real, and its eigenvectors form an orthonormal and complete basis. Furthermore, because $\Sigma$ and $M$ are related by a similarity transformation, they have identical spectra. Their eigenvectors are also simply related. Denoting the eigenvectors of $\Sigma$ by $v^{(\ell)}, \ell=1, \ldots, N$, with eigenvalues $\lambda_{\ell}$, those of $M$ are given by $W^{1 / 2} v^{(\ell)}$ with the same eigenvalues. In particular, the zero-modes are related by $v_{M}^{(1)}=W^{1 / 2} v^{(1)}$. 
In terms of $\Sigma$, the solution 17 for $P$ becomes

$$
P(t)=W^{1 / 2} \mathrm{e}^{\Sigma t} W^{-1 / 2}=W^{1 / 2}\left(v^{(1)} v^{(1) \top}+\sum_{\ell=2}^{N} \mathrm{e}^{\lambda_{\ell} t} v^{(\ell)} v^{(\ell) \top}\right) W^{-1 / 2} .
$$

As $t \rightarrow \infty$, only the zero-mode contributes:

$$
P(t \rightarrow \infty)=W^{1 / 2} v^{(1)} v^{(1) \top} W^{-1 / 2} .
$$

It is straightforward to derive an explicit expression for the zero-mode. First note that the vector with unit entries, $\vec{e} \equiv(1,1, \ldots, 1)$, is a zero-eigenvector of $Z$, that is, $Z \vec{e}=0$. It follows from 28$)$ that $\Sigma\left(W^{1 / 2} \vec{e}\right)=0$, hence $v^{(1)} \sim W^{1 / 2} \vec{e}$. Normalizing, we obtain

$$
v^{(1)}=\left(\sqrt{\frac{w_{1}}{w}}, \sqrt{\frac{w_{2}}{w}}, \ldots, \sqrt{\frac{w_{N}}{w}}\right) ; \quad w \equiv \sum_{i}^{N} w_{i} .
$$

The corresponding zero-mode of $M$ is $v_{M}^{(1)}=W^{1 / 2} v^{(1)}$. Normalizing again, we find

$$
v_{M}^{(1)}=\left(\frac{w_{1}}{w}, \frac{w_{2}}{w}, \ldots, \frac{w_{N}}{w}\right) .
$$

In other words, $P_{i j}(t \rightarrow \infty)=\frac{w_{i}}{w} e_{j}$, which gives the equilibrium distribution:

$$
f_{i}^{\infty}=\frac{w_{i}}{w} .
$$

Explicitly, using (24), we have [62, 63]:

$$
f_{i}^{\infty}=\frac{H_{i}^{3} \mathcal{N}_{i}^{-1} \mathrm{e}^{S_{i}}}{\sum_{j} H_{j}^{3} \mathcal{N}_{j}^{-1} \mathrm{e}^{S_{j}}} .
$$

Famously this distribution depends on the choice of time, which is one aspect of the measure problem. Nevertheless the dependence enters as a mild power-law correction - the distribution exponentially favors low-lying vacua, i.e., those with highest $\mathrm{dS}$ entropy.

\subsection{Downward approximation}

In our analysis we will need the complete spectrum $\lambda_{\ell}$ of the transition matrix. This exact spectrum requires diagonalizing an $N \times N$ matrix, which is computationally intensive for large $N$. A useful and intuitive approach is the "downward" approximation 90, 91, which neglects upward transitions. To see how this is justified, recall the detailed balance condition (25). Importantly, this relation only depends on the false and true vacuum potential energy. It does not depend on the potential barrier, nor does it not rely on the thin-wall approximation. The upshot is that the rate for "upward" CDL tunneling between two vacua is exponentially suppressed relative to the downward rate. The downward approximation amounts to neglecting all upward transitions to leading order.

It is convenient to label dS vacua in order of increasing potential energy, $0<V_{1} \leq V_{2} \leq \ldots \leq V_{N}$. In this case the transition matrix becomes upper-triangular, hence its eigenvalues are simply given by the diagonal elements:

$$
\lambda_{j} \simeq M_{j j}=-\kappa_{j} \quad \text { (downward approximation) },
$$

where $\kappa_{j} \equiv \sum_{r} \kappa_{r j}$ is the total rate out of vacuum $j$. The lower-triangular matrix encodes upward transitions and can be included as a perturbative correction [90, 91. To leading order in the approximation, the lowestenergy vacuum, $V_{1}$, is necessarily stable, i.e., $\kappa_{1}=0$. This sets the zero-mode. There may be other vacua in the region whose only allowed transitions involve up-tunneling. Such vacua would also become approximately stable to leading order in the downward approximation. In other words, their decay rate is subleading in downward perturbation theory. 


\subsection{Initial conditions}

Since all but a measure zero of watchers will sample a finite number of bubbles before entering a terminal vacuum, our approach, like other local measures, is sensitive to initial conditions. A possible argument, originally advocated in the context of the causal diamond measure [64, 65], is that the theory of initial conditions is in principle distinct from the measure problem and should be provided by the theory of quantum gravity. In our case, while we cannot claim complete insensitivity, we would nevertheless like to argue that our scenario is largely insensitive to initial conditions under certain assumptions.

Specifically, we suppose that our watcher starts out in a highly-perched initial dS vacuum in the bulk of moduli space, with Planckian or string-scale energy density. The decay rate of this incipient vacuum is also assumed to be near the fundamental scale, but long-lived enough to trigger eternal inflation. We further assume that the initial vacuum is surrounded by similarly Planckian/short-lived vacua, such that diffusion from the initial node proceeds rapidly, within a few Planck/string times.

As mentioned in the Introduction, our ensemble of watchers is generated by following all future "decohered" classical histories of the watcher 69. This gives an ensemble of world-lines weighted unambiguously by the quantum-mechanical branching ratios. Given our assumptions, the different watcher ${ }^{15}$ will therefore rapidly disperse and reach different regions near the boundary of moduli space, where low-lying, long-lived vacua presumably reside [151. These different, distant regions correspond to our ensemble of foraging sites. Thus, within a short time, watchers access a large number of regions (including optimal ones), and these constitute our ensemble of regions. More precisely, if we imagined coarse-graining the Markov process over large regions of the landscape, the required time would be the sampling time for the coarse-grained random walk. While the subject of coarse-grained (or "lumped") Markov processes has been extensively studied (see [89]), without detailed knowledge of the string landscape it would seem futile to estimate the coarsegrained sampling time. It is reasonable, however, that it ought to be vastly shorter than the (fine-grained) exponentially-long global mixing time for the landscape.

Once a watcher reaches a given region of the landscape, the average time needed to access any target node in the region randomly-picked according to the stationary distribution is given by the average MFPT, otherwise known as Kemeny's constant [89. As we will review in Sec. 3, a remarkable (and counterintuitive) property of Kemeny's constant is that it is independent of the starting node. In other words, the average time required to reach any node in the region is independent of the watcher's initial conditions in that region. In optimal regions, in particular, the relative occupational probabilities of the watcher will rapidly reach their (local) stationary distribution (34), thanks to the healthy transition rates between vacua in such regions. Thus, as long as there is sufficient time for optimal regions to be accessed, the Markovian dynamics will efficiently drive the occupational probabilities within these regions towards local equilibrium.

\section{First-Passage Processes}

A popular measure of random search efficiency is the mean first-passage time (MFPT) [85], defined as the average time for a random walk to reach a target for the first time. The MFPT has been used in a wide range of contexts, e.g., [82, 86 88, though to our knowledge this is the first application in the context of string landscape dynamics.

Our starting point is the "survival" probability $S_{k i}(t)$, defined as the probability that a random walker starting at node $i$ at $t=0$ has not reached site $k$ by time $t$. An important related concept is the first-passage probability density, $F_{k i}(t)$, which represents the probability density that node $k$ is being visited for the first

\footnotetext{
${ }^{15}$ To simplify the discussion, we now refer to the different branches of the watcher simply as "different" watchers.
} 
time at time $t$, given that the walker started at $i$ at $t=0$. It is related to the survival probability $S_{k i}(t)$ by

$$
S_{k i}(t)=1-\int_{0}^{t} \mathrm{~d} t^{\prime} F_{k i}\left(t^{\prime}\right) .
$$

It follows that $F_{k i}(t)=-\mathrm{d} S_{k i} / \mathrm{d} t$. For a connected network with finite $N$ number of nodes, the system is guaranteed to hit any target site given sufficient time: $S_{k i}(t) \rightarrow 0$, equivalently $F_{k i}(t) \rightarrow 1$, as $t \rightarrow \infty$. This is intimately related to the recurrence property of random walks on such networks, which will be the subject of Sec. 4. Note that we defined $F_{k i}$ as the probability density that one starts at node $i$ and ends at node $k$ at time $t$. One can alternatively consider a conditional first passage probability $F_{k i}^{(0)}(t)$ defined as the probability density that one leaves the node $i$ at $t=0$ and ends at $k$ at time $t$. The two quantities are simply related with each other. See the Appendix for further discussion.

The mean first-passage time (MFPT) is defined as the first moment of $F_{k i}(t)$ :

$$
\left\langle t_{i \rightarrow k}\right\rangle=\int_{0}^{\infty} \mathrm{d} t t F_{k i}(t)=-\left.\frac{\mathrm{d} \tilde{F}_{k i}(s)}{\mathrm{d} s}\right|_{s=0},
$$

where $\tilde{F}_{k i}(s)=\int_{0}^{\infty} \mathrm{d} t F_{k i}(t) \mathrm{e}^{-s t}$ is the Laplace transform. This represents the average time taken by a random walker to reach $k$, starting from $i$ at $t=0$, averaged on all paths connecting the two nodes. The global MFPT, otherwise known as Kemeny's constant [89, is the average time taken by a random walker to reach a target node randomly-picked according to the stationary distribution:

$$
t_{\mathrm{MFPT}} \equiv \sum_{k}\left\langle t_{i \rightarrow k}\right\rangle f_{k}^{\infty} .
$$

It is well-known that, remarkably, this quantity is independent of the starting node $i$. Another classic result of random walk theory is that Kemeny's constant can be neatly expressed as a spectral sum over the non-zero eigenvalues of the transition matrix:

$$
t_{\mathrm{MFPT}}=\sum_{\ell=2}^{N} \frac{1}{\left|\lambda_{\ell}\right|} .
$$

For completeness, we briefly review how (39) is derived for weighted random walks [150]. For this purpose it is convenient to work with discretized time, defined by $t=n \Delta t$, where $n$ is an integer. A well-known and important relation between first-passage and occupational probabilities is [85]

$$
P_{k i}(n)=\delta_{k i} \delta_{n 0}+\sum_{m=0}^{n} F_{k i}(n-m) P_{k k}(m) \Delta t
$$

where we have dropped corrections of $\mathcal{O}\left((\Delta t)^{2}\right)$. This relation is easy to understand. Aside from the obvious initial Kronecker delta, the occupational probability at the $n^{\text {th }}$ time step is given by the sum of all first-passage probabilities to $k$ at earlier times $n-m$, multiplied by the "loop" probability $P_{k k}(m)$ that the walker started and returned to $k$ in $m$ time steps. Taking the discrete Laplace transform, defined as $\tilde{P}_{k i}(s)=\sum_{n=0}^{\infty} P_{k i}(n) \mathrm{e}^{-s n \Delta t} \Delta t$, gives

$$
\tilde{P}_{k i}(s)=\delta_{k i} \Delta t+\tilde{F}_{k i}(s) \tilde{P}_{k k}(s) .
$$

The first-passage probability for $i \neq k$ (also known as the 'first-hitting' probability) is thus

$$
\tilde{F}_{k i}(s)=\frac{\tilde{P}_{k i}(s)}{\tilde{P}_{k k}(s)} .
$$


Using (29), the Laplace transform of the occupational probability is given by

$$
\tilde{P}_{k i}(s)=\frac{1}{s} f_{k}^{\infty}+\sum_{\ell=2}^{N} \frac{1}{s-\lambda_{\ell}} v_{k}^{(\ell)} v_{i}^{(\ell)} \sqrt{\frac{f_{k}^{\infty}}{f_{i}^{\infty}}} .
$$

Substituting into 42 , we obtain

$$
\tilde{F}_{k i}(s)=\frac{f_{k}^{\infty}+\sum_{\ell=2}^{N} \frac{s}{s-\lambda_{\ell}} v_{k}^{(\ell)} v_{i}^{(\ell)} \sqrt{\frac{f_{k}^{\infty}}{f_{i}^{\infty}}}}{f_{k}^{\infty}+\sum_{\ell=2}^{N} \frac{s}{s-\lambda_{\ell}} v_{k}^{(\ell) 2}} .
$$

Differentiating with respect to $s$ and setting $s=0$ gives the MFPT (37) from $i$ to $k$ :

$$
\left\langle t_{i \rightarrow k}\right\rangle=-\left.\frac{\mathrm{d} \tilde{F}_{k i}(s)}{\mathrm{d} s}\right|_{s=0}=\frac{1}{f_{k}^{\infty}} \sum_{\ell=2}^{N} \frac{1}{\left|\lambda_{\ell}\right|}\left(v_{k}^{(\ell) 2}-v_{k}^{(\ell)} v_{i}^{(\ell)} \sqrt{\frac{f_{k}^{\infty}}{f_{i}^{\infty}}}\right) .
$$

Using the orthonormality conditions $\sum_{k} v_{k}^{(\ell) 2}=1$ and $\sum_{k} v_{k}^{(\ell \neq 1)} \sqrt{f_{k}^{\infty}} \sim \sum_{k} v_{k}^{(\ell \neq 1)} v_{k}^{(1)}=0$, the average MFPT (38) is readily obtained

$$
t_{\mathrm{MFPT}}=\sum_{k}\left\langle t_{i \rightarrow k}\right\rangle f_{k}^{\infty}=\sum_{\ell=2}^{N} \frac{1}{\left|\lambda_{\ell}\right|} \text {. }
$$

This establishes 39 .

For our purposes it will be convenient to work with a dimensionless average MFPT, defined as

$$
\mathcal{T}_{\mathrm{MFPT}} \equiv \frac{t_{\mathrm{MFPT}}}{\Delta t}=\sum_{\ell=2}^{N} \frac{1}{\left|\lambda_{\ell}\right| \Delta t}
$$

This quantity is time-reparametrization invariant and counts the number of time steps taken by a random walker. It is most intuitive and easiest to calculate in the downward approximation, where the eigenvalues are given by 35$)$ :

$$
\mathcal{T}_{\text {MFPT }} \simeq \sum_{j=2}^{N} \frac{1}{\kappa_{j} \Delta t} .
$$

Thus the average MFPT is equal to the sum over the characteristic number of waiting time steps, $\left(\kappa_{i} \Delta t\right)^{-1}$, for all metastable vacua in the region. To obtain this result, we have followed the downward convention of labeling the vacua in order of increasing potential energy, as described in Sec. 2.2. In particular, the sum excludes the lowest-energy vacuum, $j=1$, which is necessarily stable in the downward approximation. Importantly, since $\kappa_{i}$ is the decay rate per unit time $t$, the dimensionless MFPT is manifestly time-reparametrization invariant, i.e., independent of the choice of time variable $t$.

A typical region in the landscape will, generically, include multiple metastable vacua whose only possible transitions involve up-tunneling. This is sketched in Fig. 2(a). To leading order in the downward approximation, such vacua become absolutely stable $\left(\kappa_{j} \sim 0\right)$, implying a divergent average MFPT (2). To get a finite result, one must work to next order in the downward approximation, but it is clear that the result will be an exponentially-long MFPT. This corresponds to frustrated dynamics. A watcher in such a region has an inefficient foraging strategy and is overwhelmingly likely to tunnel to an AdS (or Minkowski) terminal and die well before accessing a hospitable vacuum.

In the vastness of the landscape, there ought to be rare but optimal regions, whose vacua all have allowed downward transitions (except of course for the lowest-lying vacuum). Thus their topography is that of a broad energy valley or funnel, as shown in Fig. 2(b). This is akin to the principle of minimal frustration of 
protein energy landscapes [95, 96, where the high-energy unfolded states are connected to the lowest-energy native state by a relatively smooth funnel. Watchers in such regions have an efficient foraging strategy and find resources (hospitable vacua) before dying in a terminal vacuum. Thus our mechanism can be interpreted as natural selection of watchers, with 'fitness' defined as short average MFPT relative to the characteristic decay time into terminals.

Another instance of funnel topography occurs in the loss/optimization landscape of deep neural networks. In general, finding the global minimum of the loss landscape is an NP-complete problem [99. Recently it has been shown that neural networks with poor training parameters correspond to highly non-convex landscapes, with many local minima, whereas well-trained networks have smooth, funnel-like landscapes. Relatedly, the connectivity matrix of well-trained, state-of-the-art deep neural networks have power-law spectral density [101, well-described by heavy-tailed random matrix theory [102, indicating that well-trained networks operate at criticality.

To minimize the MFPT (48), downward transitions should be as fast as possible. However, as we will see in Sec. 4. transition rates are bounded from above by demanding efficient sampling of local regions, since high transition rates can cause a watcher to escape an efficient region. These considerations will lead us to define a dimensionless mean residency time. We will find that the mean residency time grows polynomially in $D=\log N$, specifically as $\log N$ in the optimal case. Incidentally this does not contradict the NP-hard complexity classification 73. NP-hardness is a worst-case assessment, and as such does not preclude the existence of polynomial-time solutions for special, finely-tuned instances of the problem.

As a toy example of how such a phase transition in complexity can occur in special instances of a problem, let us consider a landscape which has the topology of a tree [112. By this we mean that every vacuum in the landscape is connected to $b$ other vacua, so that there is one vacuum at depth zero, $b$ vacua at depth one, $b^{2}$ vacua at depth two and so on. By connectedness, we mean that each vacuum has a finite rate $\kappa_{d \rightarrow d+1}$ of transition to one of its $b$ descendants, where we have assumed for simplicity that the transition rate only depends on the depth in the tree. The total rate of transition out of a vacuum at depth $d$ is $\kappa_{d}=b \kappa_{d \rightarrow d+1}$. The average MFPT is therefore given by

$$
t_{\mathrm{MFPT}}=\sum_{d=0}^{D-1} \frac{b^{d}}{b \kappa_{d \rightarrow d+1}} .
$$

As a further simplification, let us assume that $\kappa_{d \rightarrow d+1} \sim z^{-d}$ for some parameter $z$. This then gives

$$
t_{\mathrm{MFPT}}=\frac{1-(b z)^{D}}{b(1-b z)} .
$$

Note that the MFPT is controlled by the effective parameter $p=b z$. When $p>1$, the MFPT grows exponentially with the depth, $t_{\mathrm{MFPT}} \sim \mathrm{e}^{D \ln p}$; when $p=1$, we have linear growth with depth, $t_{\mathrm{MFPT}} \sim D$; while for $p<1$ the MFPT is $\mathcal{O}(1)$ in the large $D$ limit.

\section{Recurrent and Transient Random Walks}

Up to this point we have treated the regions in our ensemble as closed systems, ignoring the exchange of probability with their surroundings. In this idealized framework, we found that the MFPT (48) can in principle be made arbitrarily small by dialing up transition rates. More realistically, however, we should treat the regions as open systems, allowing the possibility that a watcher escapes a region before hitting a target vacuum. This introduces a trade-off. Once a random walker lands in an optimal region, the dynamics should be such that the walker efficiently explores the region, thereby minimizing the MFPT, while at the same time minimizing the likelihood of escape before finding viable vacua. 
Instead of treating regions as open systems, which would require modeling their environment, we focus on the more tractable (and less model-dependent) problem of random walk dynamics in a given region in the limit of infinite volume, $N \rightarrow \infty$. To be clear, our model remains unchanged - an ensemble of many regions, each with finite $N$ nodes. However, to gain insights on the impact of their environments, we consider the behavior of random walks in the hypothetical $N \rightarrow \infty$ limit.

In this limit we demand that random walks be recurrent, i.e., that random walkers will return to their starting point, and will do so infinitely-many times in the future, with unit probability. Equivalently, in recurrent walks every site will be visited with probability one. Conversely, transient walks are such that the probability of never returning to the starting point is finite. Although not formally equivalent to modeling regions as open systems, we advocate recurrence as a reliable and model-independent benchmark for efficient sampling. Recurrent walks thoroughly explore any region around their starting point, whereas transient walks tend to escape to infinity.

\subsection{Recurrence condition}

Recall the survival probability $S_{k i}(t)$, defined in Sec. 3 as the probability that a random walker starting at node $i$ at $t=0$ has not reached site $k$ by time $t$. It follows that $S_{i i}(t)$ is the probability that the walker has not returned to the starting node by time $t$. The escape probability is naturally defined as the probability that the walker never returns to the starting node (see Appendix 7 for further discussion):

$$
\text { Escape probability }=\lim _{t \rightarrow \infty} S_{i i}(t) \text {. }
$$

A random walk is said to be recurrent if the escape probability vanishes; it is said to be transient if the escape probability is finite. That is,

$$
\begin{array}{llll}
\lim _{t \rightarrow \infty} S_{i i}(t) & =0 & \Longleftrightarrow & \text { recurrence } \\
\lim _{t \rightarrow \infty} S_{i i}(t) & =\text { finite } & \Longleftrightarrow & \text { transience }
\end{array}
$$

This criterion can be expressed in terms of the first-passage probability density $F_{k i}(t)$, defined in (36) as the probability density that a random walk starting at $i$ at $t=0$ reaches $k$ for the first time at time $t$. Thus $F_{i i}(t)$ is naturally interpreted as the first-return probability density. Using (36), we see that the escape probability satisfies

$$
\lim _{t \rightarrow \infty} S_{i i}(t)=1-\int_{0}^{\infty} \mathrm{d} t F_{i i}(t)=1-\tilde{F}_{i i}(0),
$$

where $\tilde{F}_{i i}(0)=\int_{0}^{\infty} \mathrm{d} t F_{i i}(t)$ is the probability that the random walker ever returns to $i$. It follows that

$$
\begin{array}{lll}
\tilde{F}_{i i}(0)=1 & \Longleftrightarrow & \text { recurrence } \\
\tilde{F}_{i i}(0)<1 & \Longleftrightarrow & \text { transience }
\end{array}
$$

In other words, recurrence implies that a random walker is certain to return eventually to the starting node, and will do so infinitely-many times in the future ${ }^{16}$ It is worth emphasizing that $\tilde{F}_{i i}(0)=-\int_{0}^{\infty} \mathrm{d} t \frac{\mathrm{d} S_{i i}}{\mathrm{~d} t}$ is time-reparametrization invariant. Thus the recurrence criterion is independent of the choice of time variable.

The recurrence/transience criterion (54) can be related to the occupational probability $P_{k i}(t)$. Setting $k=i$ in (41) gives

$$
\tilde{F}_{i i}(s)=1-\frac{\Delta t}{\tilde{P}_{i i}(s)},
$$

\footnotetext{
${ }^{16}$ Paradoxically, it possibly takes, on average, an infinite time to do so on an infinite network, as the mean firstreturn time $\left\langle t_{i \rightarrow i}\right\rangle=\int_{0}^{\infty} \mathrm{d} t t F_{i i}(t)$ can potentially diverge.
} 
where $\Delta t$ is a discrete time step. Thus the recurrence criterion (54) is equivalent to

$$
\mathcal{R}_{i} \equiv \lim _{s \rightarrow 0} \frac{\tilde{P}_{i i}(s)}{\Delta t}=\infty \quad \text { (recurrence) }
$$

Averaging over all initial sites, we define

$$
\mathcal{R} \equiv \lim _{s \rightarrow 0}\left\langle\frac{\tilde{P}_{i i}(s)}{\Delta t}\right\rangle=\lim _{s \rightarrow 0} \frac{\operatorname{Tr} \tilde{P}(s)}{N \Delta t}
$$

The recurrence criterion amounts to demanding that $\mathcal{R}$ diverges. The factor of $\Delta t$ has been kept for convenience, to make manifest the fact that $\mathcal{R}$ is time-reparametrization invariant. This will play an important role in what follows.

The trace of the occupational probability follows readily from 43,

$$
\frac{\operatorname{Tr} \tilde{P}(s)}{N}=\frac{1}{N s}+\frac{1}{N} \sum_{\ell=2}^{N} \frac{1}{s-\lambda_{\ell}},
$$

where we have used (13) and the normalization condition $\sum_{i} v_{i}^{(\ell) 2}=1$. At fixed $N$, the first term, which arises from the zero-mode, diverges as $s \rightarrow 0$. Hence random walks are always recurrent on finite networks. To study recurrence on infinite networks, one should first let $N \rightarrow \infty$ before sending $s \rightarrow 0$. In this limit the zero-mode contribution vanishes [152, leaving us with

$$
\mathcal{R}=\frac{1}{N} \sum_{\ell=2}^{N} \frac{1}{\left|\lambda_{\ell}\right| \Delta t},
$$

where the limit $N \rightarrow \infty$ is understood. Note that $\mathcal{R}$ can be expressed in terms of the dimensionless average MFPT (47) as

$$
\mathcal{R}=\frac{\mathcal{T}_{\mathrm{MFPT}}}{N} .
$$

Therefore, we may think of $\mathcal{R}$ as a dimensionless mean residency time.

Once again the result is most transparent in the downward approximation, where the eigenvalues are given by (35), and the MFPT reduces to (48):

$$
\mathcal{R} \simeq\left\langle\frac{1}{\kappa_{i} \Delta t}\right\rangle \quad \text { (downward) }
$$

Since $\kappa_{i}$ is the decay rate per unit time $t$, this makes the time-reparametrization invariance of $\mathcal{R}$ manifest. In particular, it will be convenient to express the result in terms of proper time: $\kappa_{i} \Delta t=\kappa_{i}^{\text {proper }} \Delta \tau_{i}$. The natural proper time step is of course the Hubble time: $\Delta \tau_{i}=H_{i}^{-1} \sim \frac{M_{\mathrm{P1}}}{\sqrt{V_{i}}}$. Putting everything together,

$$
\mathcal{R} \simeq\left\langle\frac{\sqrt{V_{i}}}{M_{\mathrm{Pl}} \kappa_{i}^{\text {proper }}}\right\rangle=\left\langle\frac{\sqrt{V_{i}}}{M_{\mathrm{Pl}}} \tau_{i}\right\rangle
$$

where $\tau_{i} \equiv 1 / \kappa_{i}^{\text {proper }}$ is the (proper) lifetime of the $i^{\text {th }}$ vacuum. Whether (62) is finite or diverges as $N \rightarrow \infty$ determines whether the random walk is transient or recurrent, respectively ${ }^{17}$

\footnotetext{
${ }^{17}$ To qualify as a semiclassical vacuum, a given vacuum should at least survive for a Hubble time, i.e., $H_{i} \tau_{i} \gtrsim 1$. It follows that $\mathcal{R} \gtrsim 1$. Using (59), we learn that $\mathcal{T}_{\text {MFPT }} \gtrsim \mathcal{O}(N)$, that is, the MFPT must scale at least linearly in $N$.
} 


\subsection{Statistical average}

Our next task is to express 62 as a suitable statistical average over possible realizations of the region. In general the rate $\kappa_{i}$ out of a given metastable vacuum depends on its potential energy $V_{i}$ as well as the shape of the potential barriers that determine the bounce solution to the different destination vacua. Denoting the "shape" parameters for transitions out of the $i^{\text {th }}$ vacuum collectively by $\theta_{i}$, we have

$$
\kappa_{i}^{\text {proper }} \equiv \kappa^{\text {proper }}\left(V_{i}, \theta_{i}\right)
$$

The dependence on $V_{i}$ comes from two sources. An immediate source is the volume factor $H_{i}^{-3} \sim V_{i}^{-3 / 2}$ of de Sitter false vacuum. A second, and less obvious factor comes from the expected suppression of possible destination vacua as $V_{i} \rightarrow 0$. Indeed, the assumption of a flat probability distribution of vacuum energy and the exponential suppression for upward tunneling together imply that, statistically, lower-lying vacua have fewer possible dS destinations than high-lying vacua.

Let $\mathcal{P}(V, \theta)$ denote the underlying joint probability distribution for a given vacuum to have potential energy $V$ and bounce parameters $\theta$. In the large $N$ limit the dimensionless mean residency time can be approximated via the central limit theorem by a statistical average weighted by the underlying probability distribution:

$$
\mathcal{R}=\int \mathrm{d} V \mathrm{~d} \theta \frac{\sqrt{V}}{M_{\mathrm{Pl}}} \tau(V, \theta) \mathcal{P}(V, \theta),
$$

with corrections suppressed by $1 / \sqrt{N}$. For simplicity let us assume that on the string landscape the absolute height of a vacuum and the shape of the surrounding potential barriers are uncorrelated:

$$
\mathcal{P}(V, \theta) \equiv \mathcal{P}(V) \hat{\mathcal{P}}(\theta)
$$

Equation 64 then simplifies to

$$
\mathcal{R}=\int \mathrm{d} V \frac{\sqrt{V}}{M_{\mathrm{Pl}}} \tau(V) \mathcal{P}(V)
$$

where

$$
\tau(V) \equiv \int \mathrm{d} \theta \tau(V, \theta) \hat{\mathcal{P}}(\theta)
$$

is the average lifetime of a vacuum with potential energy $V$.

Not surprisingly, the recurrence criterion $\mathcal{R} \rightarrow \infty$ is sensitive to the behavior of $\tau(V)$ for the lowest-lying (and, as argued above, generically most stable) vacua. Provided the probability distribution $\mathcal{P}(V)$ falls off sufficiently fast for large $V$, the divergence in $\mathcal{R}$ must come from the small- $V$ region of the integral. Therefore, random walks will be recurrent if the average lifetime $\tau(V)$ diverges sufficiently fast as $V \rightarrow 0$. Specifically, assuming as before that $\mathcal{P}(V)$ is nearly flat for $V$ much smaller than the fundamental scale, the recurrence criterion is

$$
\mathcal{R} \sim \int_{0} \mathrm{~d} V \sqrt{V} \tau(V) \rightarrow \infty \quad \text { (recurrence) }
$$

This condition will be satisfied if $\tau(V)$ diverges faster than $V^{-3 / 2}$ as $V \rightarrow 0$, with the critical case $\sim V^{-3 / 2}$ resulting in a logarithmic divergence.

\section{Dynamical Criticality}

We have derived two competing conditions: minimal dimensionless average MFPT, which as argued in Sec. 3 requires fast transition rates; and recurrence in the $N \rightarrow \infty$ limit, which as shown in Sec.4 4 requires sufficiently long-lived vacua. Optimal regions reach a compromise: they achieve the shortest MFPT compatible with 
recurrence, i.e., the least-divergent recurrence integral (68). Thus, optimal regions lie at the critical boundary between recurrent and transient walks, characterized by an average lifetime scaling as $V^{-3 / 2}$. Using the Planck mass $M_{\mathrm{Pl}}$ to fix dimensions, since this is the only other scale at hand, we obtain

$$
\tau_{\text {crit }}(V) \sim \frac{M_{\mathrm{Pl}}^{5}}{V^{3 / 2}} \sim \frac{M_{\mathrm{Pl}}^{2}}{H^{3}} \quad \text { as } V \rightarrow 0 .
$$

Remarkably, this is recognized as the Page time [104 for dS space [105 108. In slow-roll inflation, the Page time marks the phase transition to slow-roll eternal inflation [109] and has been used to place a bound on the maximum number of e-folds that can be described semi-classically [110. The appearance of the Page time in false-vacuum eternal inflation, and its relation to random walk criticality, is surprising. We do not yet have a compelling intuitive explanation for its occurence.

In any case, the critical lifetime 69 implies a logarithmically-divergent integral 68$) 18$ Correspondingly, at finite $N$ the mean residency time (62) diverges as $\log N$, which signals dynamical criticality. A similar non-equilibrium phase transition occurs in quenched disordered media, when the probability distribution for waiting times reaches a critical power-law 93. Thus the joint demands of sweeping exploration, defined by recurrence, and minimal oversampling, defined by minimal MFPT, select regions of the landscape that are tuned at criticality 19

The criticality of the recurrence/transience boundary can be understood in various ways. From a computational complexity standpoint, it represents a transition in the scaling of the mean residency time (62) as a function of the effective dimensionality $D$ of the landscape region. (Recall that the number of vacua scales exponentially with the landscape dimension, $N \sim \mathrm{e}^{D}$.) Recurrent walks typically result in a power-law divergent integral in (68), corresponding at finite $N$ to a recurrence measure diverging exponentially in $D$. In the critical case, the recurrence measure instead grows logarithmically in $N$, and therefore polynomially in $D$. More precisely, the large $N$ behavior delineates regions in the landscape into three basic types:

$$
\mathcal{R} \sim\left\{\begin{array}{ccc}
\operatorname{Exp}(D) & \cdots & \text { (recurrent but high complexity) } \\
\operatorname{Poly}(D) & \cdots & \text { (recurrent and low complexity) } \\
\text { Constant } & \cdots & \text { (low complexity but transient) }
\end{array}\right.
$$

The second line corresponds to a critical boundary between the other two phases. The phase transition we have uncovered belongs in the category of computational phase transitions, which also occur in the infinitesize limit. These have generated much activity in computer science in recent years [111, 112. A famous example is the phase transition in heuristic decision-tree pruning from polynomial to exponential search time at a critical value of the effective branching ratio [154. Another example is the phase transition from satisfiability to unsatisfiability in the random $k$-SAT problem at a critical value of the ratio of constraints to variables [155].

The recurrence/transience boundary is also critical in that it delineates stable and unstable dynamical behavior. To see this, consider a pair of random walks starting from the same initial site. In the recurrent regime, the walkers are certain to meet again, infinitely-many times, in the future. In the transient regime, however, they will eventually diverge from each other, never to meet again. Thus the pair of walks can be thought of as dynamically stable in the recurrent case, and unstable in the transient case.

\footnotetext{
${ }^{18}$ Equation 69 captures the leading divergence as $V \rightarrow 0$. We cannot constrain slowly-varying factors, such as $V^{-3 / 2} \log ^{n} V$, which, in any case, give negligible corrections to the phenomenological predictions discussed in Sec. 6 .

${ }^{19}$ There is a simple analogue in the case of simple (Brownian) random walks on regular $d$-dimensional lattices. For fixed number of sites $N$, the average MFPT decreases with increasing dimension. For instance, $t_{\mathrm{MFPT}}=N(N+1) / 6$ for $d=1, t_{\mathrm{MFPT}}=\pi^{-1} N \ln N+\mathcal{O}(N)$ for $d=2$, and $t_{\mathrm{MFPT}} \simeq 1.52 N+\mathcal{O}(\sqrt{N})$ for $d=3[153$. On the other hand, by Pólya's theorem simple random walks are recurrent for $d \leq 2$, and transient for $d>2[103$. The minimal MFPT compatible with recurrence is achieved in $d=2$, the critical dimension for recurrence/transience.
} 
As a proof of principle, the desired critical scaling $(69)$ can arise in the following simplistic situation. Consider a funnel-like region of the landscape with effective dimensionality $D$. Suppose that the potential energy is described by a smooth power-law envelope $V(\rho) \sim \rho^{n}$, where $\rho$ is the distance in moduli space, punctuated by a lattice of metastable vacua that are equally spaced on average. Thus within a given distance $R$ there are $\sim R^{D}$ vacua. Furthermore, suppose that each vacuum in the funnel can transition to all lower vacua with approximately equal rate ${ }^{20}$ Then the total transition rate out of a given vacuum with potential energy $V_{j} \sim R_{j}^{n}$ is proportional to the number of destinations $\sim R_{j}^{D} \sim V_{j}^{D / n}$. This must be divided by $H_{j}^{3} \sim V_{j}^{3 / 2}$ to obtain the proper total transition rate: $\kappa_{j}^{\text {proper }} \sim V^{\frac{D}{n}-\frac{3}{2}}$. This gives the desired scaling (69) for $D=3 n$.

In the natural world there is a striking relation between complexity of self-organizing systems and criticality [133. Examples include neuronal activity in the brain, flocking behavior of starlings, insect swarms and cell growth, to name a few. Such systems operate in a critical state between stable and unstable dynamical regimes. Empirical observations that support the dynamical criticality hypothesis include, for instance, the probability distribution of neuronal avalanche size being scale-free [131, and correlation functions of velocity dispersion of flocking birds displaying scale invariance [136. It has been conjectured that dynamical criticality is evolutionarily favored because it offers an ideal compromise between robust response to external stimuli and flexibility for adaptation to environmental changes. Similarly, our mechanism offers a dynamical explanation for why our universe is poised at criticality.

\section{Phenomenological Implications}

The natural selection mechanism based on search optimization outlined above has concrete phenomenological implications for our vacuum. Importantly, these specific predictions do not rely on anthropic reasoning; instead they follow immediately from the properties of optimal regions of the landscape.

Lifetime of our vacuum: If our vacuum is part of an optimal region of the landscape, characterized by vacua with critical lifetime given the Page time $(69)$, then we predict a lifetime of

$$
\tau \sim \frac{M_{\mathrm{Pl}}^{2}}{H_{0}^{3}} \sim 10^{130} \text { years. }
$$

This explains the metastability of the electroweak vacuum. As mentioned in the Introduction, what makes the Higgs metastability particularly interesting is that it relates the observed cosmological constant, through the $H_{0}^{-3}$ volume factor, and electroweak physics, in particular the Higgs and top quark masses. Thus, taking the observed vacuum energy $\sim M_{\mathrm{Pl}}^{2} H_{0}^{2}$ as given, the optimal lifetime (71) can be interpreted as constraining the Higgs and top masses to lie around the weak scale (keeping other SM parameters fixed).

Quantitatively, the predicted lifetime agrees with the SM prediction (1) to within $\gtrsim 2 \sigma$. Closer agreement can be achieved if the top quark is slightly heavier, $m_{\mathrm{t}} \simeq 174.5 \mathrm{GeV}$. This can be viewed as a prediction, assuming of course that the SM is valid all the way to the Planck scale. New physics at intermediate scales can reduce the tension. For instance, adding a gauge-invariant, higher-dimensional operator $\frac{h^{6}}{\Lambda_{\mathrm{NP}}^{2}}$ can affect the predicted lifetime if $\Lambda_{\mathrm{NP}} \lesssim 10^{13} \mathrm{GeV}$, assuming the central value $m_{\mathrm{t}}=173.5 \mathrm{GeV}$ [17. Another possibility are sufficiently light primordial black holes which act as catalysts for the decay [157.

More generally, our mechanism selects regions with efficient transition rates, particularly for low-lying vacua, and thus gives a raison d'être for the conspiracy underlying the Higgs metastability. In other words, from our point of view the inferred metastability of the electroweak vacuum is sacred. New physics below the SM instability scale, $\sim 10^{10} \mathrm{GeV}$, on the other hand, can jeopardize this observable. Here are the implications for some candidates of BSM physics:

\footnotetext{
${ }^{20}$ This could be achieved, for instance, with 'giant leaps' [156].
} 
- Low-scale SUSY: If the SUSY breaking scale is $\lesssim 10^{10} \mathrm{GeV}$, this will directly impact the stability of our vacuum. There are three obvious possibilities: 1) SUSY makes our vacuum unstable (e.g., via decay to charge/color breaking vacua [158, 163]), which by itself is inconsistent and would therefore requires additional new physics; 2) SUSY makes our vacuum stable, which is disfavored by our mechanism; 3) SUSY maintains our vacuum within the metastability region. The latter possibility is logically consistent with our mechanism but would require further numerical conspiracy, above and beyond that already achieved in the SM. Therefore, barring fine-tunings, the natural implication of SUSY below $10^{10} \mathrm{GeV}$ is to make our vacuum stable, which is disfavored by our mechanism.

This expectation is borne out by an explicit calculation of [140, which showed that if all SUSY partners have masses at the SUSY breaking scale, then the metastability of our vacuum requires a SUSY breaking scale of $\gtrsim 10^{10} \mathrm{GeV}$. Thus optimal regions of the landscape are characterized by very high-scale SUSY breaking, which is consistent with the absence of low-scale SUSY at the LHC.

- Sterile neutrinos: Massive right-handed neutrinos, like the top quark, tend to make the vacuum less stable. Assuming three right-handed neutrinos of comparable mass, for simplicity, the impact on Higgs metastability is negligible if their mass is $\lesssim 10^{13} \mathrm{GeV}$ [139. On the other hand, if their mass is around $10^{13}-10^{14} \mathrm{GeV}$, then the expected lifetime for our vacuum will be in closer agreement with the predicted optimal lifetime (71).

- QCD axion: Consider the QCD axion as a solution to the strong CP problem. The radial part of the $U(1)$ complex scalar is a boson and hence makes the electroweak vacuum more stable. To preserve the desired metastability, the Peccei-Quinn scale must be sufficiently high, $f_{\mathrm{a}} \gtrsim 10^{10} \mathrm{GeV}[164$.

Cosmological constant problem: Unlike the above predictions, which depend only on optimality, the prediction for the cosmological constant is sensitive to the choice of measure. Assuming that vacua are weighted by comoving volume, then the local equilibrium distribution is $f_{i}^{\infty} \sim \mathrm{e}^{48 \pi^{2} M_{\mathrm{Pl}}^{4} / V_{i}}$. The distribution is sharply peaked and exponentially favors the lowest-lying vacuum in the region. Thus, within an optimal region selected by the search optimization principle, we are overwhelmingly likely to find ourselves in the vacuum with the smallest potential energy $V_{\text {min }}$ within that region.

Assuming as before that the underlying probability distribution $\mathcal{P}(V)$ is nearly flat for $V \ll M_{\mathrm{Pl}}$, then statistically the minimum potential energy achieved in a region of $N$ vacua is

$$
V_{\min } \sim \frac{M_{\mathrm{Pl}}^{4}}{N}
$$

This can account for the observed cosmological constant if our region contains $N \sim 10^{120}$ vacua. In particular, the predicted value of the cosmological constant is set by the region size $N$, which is manifestly UV-insensitive.

Because an identical argument could be made in the "global" approach to the landscape [66, 151, 165, it is important to highlight a few key differences. The global equilibrium distribution (achieved after an exponentially-long mixing time) is identical to the aforementioned local stationary distribution, thus it exponentially favors the smallest positive vacuum energy anywhere in the landscape. This cannot be considered a satisfactory solution to the cosmological constant problem, on a number of counts. (See Sec. 5 of 73 for a discussion of some of these problems.) One issue is that, from a computational complexity perspective, the problem of finding the minimal positive vacuum energy globally is harder than NP, i.e., a candidate solution cannot be checked in polynomial time. The most important issue, however, is phenomenological vacua with the smallest positive potential energies are expected to be nearly supersymmetric, with vacuum energy $\sim m_{3 / 2}^{4}$, thus the global measure would seem to overwhelmingly predict a tiny SUSY breaking scale.

These problems do not afflict the "local" approach. The optimal regions selected by our mechanism naturally have a very high SUSY breaking scale, as already mentioned. This addresses Banks' interpretation of the cosmological constant problem, namely "given the value of the cosmological constant, why is SUSY 
breaking so large?" [166. By construction the optimal regions comprise a finite number of dS vacua, and all one needs to explain the observed cosmological constant is for one of these regions to have $N \sim 10^{120}$ vacua.

\section{Conclusions}

It is striking that a number of major theoretical puzzles in fundamental physics can be interpreted as problems of near criticality. The weak hierarchy problem translates to the Higgs field being close to the phase transition between broken and unbroken electroweak symmetry [40. The cosmological constant problem is the statement that our universe is nearly flat, Minkowski space, which in turn can be thought of as a quantum critical point between dS and AdS space-times. The approximate scale invariance of primordial density perturbations suggests near criticality in the early universe. This is realized explicitly through a period of approximate dS expansion, during which the inflating universe is conformally invariant.

Yet another indication of criticality is the inferred metastability of the electroweak vacuum [11. The Higgs metastability, which is the result of a delicate numerical conspiracy, crucially relies on the absence of new physics above the weak scale. As such it leaves no room for indifference. Either one concedes that SM metastability is purely a numerical coincidence, as implicitly assumed, for instance, in all BSM theories with low-scale SUSY. Or it is a real phenomenon in want of an explanation, in which case naturalness cannot be the answer to the weak hierarchy problem.

In our mind, the near criticality of our universe strongly suggests a statistical physics origin. A natural arena for the statistical physics of universes is the landscape of string theory, together with the mechanism of eternal inflation for populating vacua. In this paper we presented a dynamical selection mechanism, based on search optimization, which favors vacua in regions of the landscape where random walk dynamics are at criticality. This suggests a connection between the near criticality of our universe and non-equilibrium critical phenomena in landscape dynamics.

Instead of concentrating on late-time stationary probability distributions, in this work we focused on the approach to equilibrium. The underlying assumption is that cosmological evolution on the multiverse has occurred for a time much shorter than the exponentially-long global mixing time of the landscape 75 . Accessing hospitable vacua then becomes a race among watchers random walking on the landscape. The "winners" are watchers that land in optimal regions of the landscape where the search algorithm is efficient.

We defined optimality by two competing requirements. The first requirement is search efficiency, quantified by the average MFPT, which requires fast transition rates. The average MFPT is the average time taken by a watcher to reach a target vacuum, picked at random according to the stationary probability distribution. We showed that the average MFPT is minimized for hospitable vacua lying at the bottom of funnel-like regions, akin to the smooth folding funnels of naturally-occurring proteins and the convex loss functions of well-trained deep neural networks. The second requirement is sweeping exploration, which requires recurrence in the infinite-volume limit. Optimal regions reach a compromise by lying at the critical boundary between recurrence and transience, thereby achieving dynamical criticality. In other words, they have the shortest MFPT compatible with recurrence. Crucially, unlike other statistical measures on the landscape, our optimality criterion (and the critical boundary it defines) is time-reparametrization invariant.

Remarkably, vacua in optimal regions have lifetimes of order the dS Page time, $\tau_{\text {crit }} \sim M_{\mathrm{Pl}}^{2} / H^{3}$. The emergence of the Page time in relation with critical dynamics on the landscape is surprising to us, and we do not yet have an intuitive explanation. In any case, for our vacuum this implies a predicted lifetime of $M_{\mathrm{Pl}}^{2} / H_{0}^{3} \sim 10^{130}$ years, which agrees with the SM prediction (1) to within $\gtrsim 2 \sigma$. Closer agreement can be achieved if the top quark is slightly heavier, $m_{\mathrm{t}} \simeq 174.5 \mathrm{GeV}$, or, more plausibly, with new physics at intermediate scales, such as $\sim 10^{13} \mathrm{GeV}$ right-handed neutrinos. Barring fine-tunings, our mechanism predicts that the SUSY breaking scale should be high, $\gtrsim 10^{10} \mathrm{GeV}$. Evidence of SUSY at the LHC would likely rule out our scenario. 
Philosophically our proposal is diametrically opposite to the standard approach to the landscape. The principle of mediocrity amounts to the statement that our vacuum should be typical among all hospitable vacua on the landscape. Instead, the hospitable vacua favored by our mechanism are exceptional, in that they live in fine-tuned neighborhoods of the landscape. But they are exceptional for a purpose - to be easily accessed early on. Our dynamical mechanism can be interpreted as natural selection of watchers foraging different regions of the landscape. Watchers with inefficient foraging strategies are likely to enter a terminal vacuum and die before finding a hospitable vacuum, while those with optimal foraging strategy are able to find hospitable vacuum early on.

Complex self-organized systems poised at criticality are ubiquitous in the natural world. This has motivated the conjecture that dynamical criticality is favored evolutionarily because it offers an ideal trade-off between robust, reproducible response and flexibility of adaptation to a changing environment. Furthermore, it has been argued that computational capabilities are maximized at criticality. Similarly, our natural selection mechanism favors vacua at criticality.

The selection mechanism presented here offers many new avenues of investigation. In this paper we have treated AdS/Minkowski vacua conservatively as terminal, acting as absorbing nodes or probability sinks. In a separate paper we will consider the more speculative possibility that collapsing AdS regions can sometimes bounce and avoid big crunch singularities, as considered in [68, 147. See also 69, 167-170. The high energy density reached at the bounce would allow the space-time region to transition classically to possibly distant $\mathrm{dS}$ vacua. Thus AdS vacua could then be interpreted as mediators of new, non-local transitions between dS vacua. We will argue that AdS-mediated transitions are analogous to Lévy flights, which have been shown to improve search efficiency [171-175]. Indeed, inspired by the saltatory behavior of foraging animals [176-180, it has been conjectured that optimal search strategies to find sparse, randomly-located targets on a network combines local (Brownian) moves with non-local (Lévy) relocations. A classic example in network science is Google's PageRank algorithm [181, 182.

The most interesting implications may be for string phenomenology and model-building. String vacua with realistic particle physics are usually considered in isolation, without consideration for their accessibility and the topography of the surrounding landscape region. In the context of flux compactifications, it has been argued that realistic vacua with small $\theta_{\mathrm{QCD}}$ (either because of a light axion or because of spontaneous breaking of strong $\mathrm{CP}$ ) or sufficiently long baryon lifetime, neither of which can be explained by anthropic arguments, are overall very rare [183. In intersecting D-brane models, it has been estimated that vacua with SM-like gauge group with three generations are one in a billion 184. It would be very interesting to explore whether such desirable phenomenological features, while rare in the realm of all possible hospitable vacua, may be more common for vacua in funnel-like regions of the landscape.

The dimensionality of space is another phenomenological issue on which our mechanism can be brought to bear. While it is plausible that life in one or two dimensions is impossible [185], arguments against more than three dimensions are less convincing. (For instance, the stability of planetary orbits does not forbid mm-size extra dimensions.) It is tempting to speculate that funnel-like regions of the landscape correspond to a low effective moduli-space dimensionality, particularly in the vicinity of the lowest-energy vacuum. Such effective reduction in dimensionality is seen in protein landscapes, where the number of conformation paths decreases dramatically near the native state. Incidentally, we have offered an explanation for the metastability of our vacuum, but why should the Higgs field be the mediator of this instability, instead of some other scalar field? A decreased effective dimensionality in our neighborhood of the landscape would explain why it is incumbent on the Higgs field to be the agent of doom.

Another obvious arena where our mechanism promises to offer new insights is the early universe, particularly inflation. At the phenomenological level, the inflationary paradigm is highly successful - a phase of approximate de Sitter expansion yields a nearly scale invariant and Gaussian spectrum of density perturbations, in excellent agreement with observations. The debate begins the moment one writes down a potential, with the usual points of contention revolving around the inflationary potential and/or initial conditions being 
unnatural/fine-tuned. A tantalizing possibility in the context of our mechanism is that slow-roll inflation with sufficient number of e-folds, and the near-critical dynamics it entails, occurs generically in funnel-like regions in the approach to the lowest-lying vacuum. Relatedly, the appearance of the Page time as the optimal lifetime vacua in our analysis is enticing and calls for a deeper understanding.

Alternatively our mechanism may suggest new ways of realizing inflation. The standard inflationary scenario is based on near-equilibrium dynamics, as the inflaton equation of motion is derivable from a local Hamiltonian. From this point of view designing a sufficiently flat potential is akin to an experimentalist dialing the temperature of an Ising spin system near criticality. In contrast, self-organized criticality, and the related notion of generic scale invariance [186, 187] are intrinsically dissipative, non-equilibrium phenomena. Within our framework it is natural to consider non-equilibrium inflationary dynamics. An optimal region is an open system, continuously exchanging probability with its environment. It will be interesting to see whether inflation could be realized as a self-organized critical phenomenon.

Acknowledgements: We warmly thank Riccardo Penco for collaboration in the early stages of this project and for many discussions. We thank Stephon Alexander, Vijay Balasubramanian, Cliff Burgess, Ulf Danielsson, Frederik Denef, Ben Freivogel, Jaume Garriga, Jonathan Heckman, Thomas Hertog, Mark Hertzberg, Lam Hui, Austin Joyce, Daniel Kabat, Tom Lubensky, Patrick Meade, Yasunori Nomura, Leonardo Senatore, Mark Trodden, Thomas Van Riet and Alex Vilenkin for helpful discussions. We are grateful to Iain Mathieson of the Penn Genetics Department for valuable discussions on natural selection. J.K. is supported in part by the US Department of Energy (HEP) Award DE-SC0013528, NASA ATP grant 80NSSC18K0694, the Charles E. Kaufman Foundation of the Pittsburgh Foundation, and a W. M. Keck Foundation Science and Engineering Grant. O.P. is supported by the Simons Foundation (\# 385592, Vijay Balasubramanian) through the It From Qubit Simons Collaboration, and the US Department of Energy contract \# FG0205ER-41367.

\section{Appendix: Alternate Definition of First Passage Probability}

In the main text we defined the first passage probability $F_{k i}(t)$ as the probability density that one starts at node $i$ at $t=0$ and ends at node $k$ at time $t$. Here we consider a slightly different version, the conditional

first passage probability $F_{k i}^{(0)}$, defined as the probability density that one leaves the node $i$ at $t=0$ and ends at $k$ within a time interval at time $t$. To see how the two quantities are related, recall 40):

$$
P_{k i}(n)=\delta_{k i} \delta_{n, 0}+\sum_{m=0}^{n} F_{k i}(n-m) P_{k k}(m) \Delta t .
$$

On the other hand, we can alternately write

$$
P_{k i}(n)=\delta_{k i} \delta_{n, 0}+\sum_{m=0}^{n} \sum_{r=0}^{n-m} R_{i i}(r) F_{k i}^{(0)}(n-m-r) P_{k k}(m) \Delta t,
$$

where $R_{i i}(r)=\left(1-\kappa_{i} \Delta t\right)^{r}$ is the probability of sticking to the node $i$ for $r$ time steps. Clearly,

$$
F_{k i}(n)=\sum_{r=0}^{n} R_{i i}(r) F_{k i}^{(0)}(n-r) .
$$

We can take a Laplace transform of 75 to obtain

$$
\tilde{F}_{k i}(s)=\frac{1}{\Delta t} \tilde{R}_{i i}(s) \tilde{F}_{k i}^{(0)}(s),
$$


where

$$
\tilde{R}_{i i}(s)=\sum_{n=0}^{\infty} R_{i i}(n) e^{-n s \Delta t} \Delta t=\frac{\Delta t}{1-\left(1-\kappa_{i} \Delta t\right) e^{-s \Delta t}} .
$$

In the $s \rightarrow 0$ limit, (76) becomes

$$
\tilde{F}_{k i}(0)=\frac{1}{\kappa_{i} \Delta t} \tilde{F}_{k i}^{(0)}(0) .
$$

The escape probability in (51) can now be written in terms of the conditional first passage probability as

$$
\lim _{t \rightarrow \infty} S_{i i}(t)=\frac{\kappa_{i} \Delta t-\tilde{F}_{k i}^{(0)}(0)}{\kappa_{i} \Delta t}
$$

We can interpret the numerator as the probability of leaving the node $i$ at $t=0$ minus the probability of leaving the node $i$ at $t=0$ and returning to $i$ at some later time. This is precisely the probability of leaving the node $i$ at $t=0$ and never returning. Therefore, if we start with some number of walkers at node $i$, the escape probability is the the ratio of the number of walkers who leave at time $t=0$ and never return to the total number of walkers who leave at time $t=0$.

\section{References}

[1] S. Weinberg, "The Cosmological Constant Problem," Rev. Mod. Phys. 61, 1 (1989).

[2] G. F. Giudice, "The Dawn of the Post-Naturalness Era," arXiv:1710.07663 [physics.hist-ph].

[3] P. H. Frampton, "Vacuum Instability and Higgs Scalar Mass," Phys. Rev. Lett. 37, 1378 (1976) Erratum: [Phys. Rev. Lett. 37, 1716 (1976)].

[4] M. Sher, "Electroweak Higgs Potentials and Vacuum Stability," Phys. Rept. 179, 273 (1989).

[5] J. A. Casas, J. R. Espinosa and M. Quiros, "Improved Higgs mass stability bound in the standard model and implications for supersymmetry," Phys. Lett. B 342, 171 (1995) [hep-ph/9409458].

[6] J. R. Espinosa and M. Quiros, "Improved metastability bounds on the standard model Higgs mass," Phys. Lett. B 353, 257 (1995) [hep-ph/9504241].

[7] G. Isidori, G. Ridolfi and A. Strumia, "On the metastability of the standard model vacuum," Nucl. Phys. B 609, 387 (2001) [hep-ph/0104016].

[8] J. R. Espinosa, G. F. Giudice and A. Riotto, "Cosmological implications of the Higgs mass measurement," JCAP 0805, 002 (2008) [arXiv:0710.2484 [hep-ph]].

[9] J. Ellis, J. R. Espinosa, G. F. Giudice, A. Hoecker and A. Riotto, "The Probable Fate of the Standard Model," Phys. Lett. B 679, 369 (2009) [arXiv:0906.0954 [hep-ph]].

[10] G. Degrassi, S. Di Vita, J. Elias-Miro, J. R. Espinosa, G. F. Giudice, G. Isidori and A. Strumia, "Higgs mass and vacuum stability in the Standard Model at NNLO," JHEP 1208, 098 (2012) [arXiv:1205.6497 [hep-ph]].

[11] D. Buttazzo, G. Degrassi, P. P. Giardino, G. F. Giudice, F. Sala, A. Salvio and A. Strumia, "Investigating the near-criticality of the Higgs boson," JHEP 1312, 089 (2013) [arXiv:1307.3536 [hep-ph]].

[12] Z. Lalak, M. Lewicki and P. Olszewski, "Higher-order scalar interactions and SM vacuum stability," JHEP 1405, 119 (2014) [arXiv:1402.3826 [hep-ph]]. 
[13] A. Andreassen, W. Frost and M. D. Schwartz, "Consistent Use of the Standard Model Effective Potential," Phys. Rev. Lett. 113, no. 24, 241801 (2014) [arXiv:1408.0292 [hep-ph]].

[14] V. Branchina, E. Messina and M. Sher, "Lifetime of the electroweak vacuum and sensitivity to Planck scale physics," Phys. Rev. D 91, 013003 (2015) [arXiv:1408.5302 [hep-ph]].

[15] A. V. Bednyakov, B. A. Kniehl, A. F. Pikelner and O. L. Veretin, "Stability of the Electroweak Vacuum: Gauge Independence and Advanced Precision," Phys. Rev. Lett. 115, no. 20, 201802 (2015) [arXiv:1507.08833 [hep-ph]].

[16] G. Iacobellis and I. Masina, "Stationary configurations of the Standard Model Higgs potential: electroweak stability and rising inflection point," Phys. Rev. D 94, no. 7, 073005 (2016) [arXiv:1604.06046 [hep-ph]].

[17] A. Andreassen, W. Frost and M. D. Schwartz, "Scale Invariant Instantons and the Complete Lifetime of the Standard Model," Phys. Rev. D 97, no. 5, 056006 (2018) [arXiv:1707.08124 [hep-ph]].

[18] G. Isidori, V. S. Rychkov, A. Strumia and N. Tetradis, "Gravitational corrections to standard model vacuum decay," Phys. Rev. D 77, 025034 (2008) [arXiv:0712.0242 [hep-ph]].

[19] A. Salvio, A. Strumia, N. Tetradis and A. Urbano, "On gravitational and thermal corrections to vacuum decay," JHEP 1609, 054 (2016) [arXiv:1608.02555 [hep-ph]].

[20] S. Weinberg, "Anthropic Bound on the Cosmological Constant," Phys. Rev. Lett. 59, 2607 (1987).

[21] V. Agrawal, S. M. Barr, J. F. Donoghue and D. Seckel, "The Anthropic principle and the mass scale of the standard model," Phys. Rev. D 57, 5480 (1998) [hep-ph/9707380].

[22] A. Kobakhidze and A. Spencer-Smith, "Electroweak Vacuum (In)Stability in an Inflationary Universe," Phys. Lett. B 722, 130 (2013) [arXiv:1301.2846 [hep-ph]].

[23] K. Enqvist, T. Meriniemi and S. Nurmi, "Generation of the Higgs Condensate and Its Decay after Inflation," JCAP 1310, 057 (2013) [arXiv:1306.4511 [hep-ph]].

[24] M. Fairbairn and R. Hogan, "Electroweak Vacuum Stability in light of BICEP2," Phys. Rev. Lett. 112, 201801 (2014) [arXiv:1403.6786 [hep-ph]].

[25] K. Enqvist, T. Meriniemi and S. Nurmi, "Higgs Dynamics during Inflation," JCAP 1407, 025 (2014) [arXiv:1404.3699 [hep-ph]].

[26] M. Herranen, T. Markkanen, S. Nurmi and A. Rajantie, "Spacetime curvature and the Higgs stability during inflation," Phys. Rev. Lett. 113, no. 21, 211102 (2014) [arXiv:1407.3141 [hep-ph]].

[27] K. Kamada, "Inflationary cosmology and the standard model Higgs with a small Hubble induced mass," Phys. Lett. B 742, 126 (2015) [arXiv:1409.5078 [hep-ph]].

[28] A. Salvio, "A Simple Motivated Completion of the Standard Model below the Planck Scale: Axions and Right-Handed Neutrinos," Phys. Lett. B 743, 428 (2015) [arXiv:1501.03781 [hep-ph]].

[29] A. Shkerin and S. Sibiryakov, "On stability of electroweak vacuum during inflation," Phys. Lett. B 746, 257 (2015) [arXiv:1503.02586 [hep-ph]].

[30] J. Kearney, H. Yoo and K. M. Zurek, "Is a Higgs Vacuum Instability Fatal for High-Scale Inflation?," Phys. Rev. D 91, no. 12, 123537 (2015) [arXiv:1503.05193 [hep-th]].

[31] J. R. Espinosa, G. F. Giudice, E. Morgante, A. Riotto, L. Senatore, A. Strumia and N. Tetradis, "The cosmological Higgstory of the vacuum instability," JHEP 1509, 174 (2015) [arXiv:1505.04825 [hep-ph]]. 
[32] D. G. Figueroa, J. Garcia-Bellido and F. Torrenti, "Decay of the standard model Higgs field after inflation," Phys. Rev. D 92, no. 8, 083511 (2015) [arXiv:1504.04600 [astro-ph.CO]].

[33] M. Herranen, T. Markkanen, S. Nurmi and A. Rajantie, "Spacetime curvature and Higgs stability after inflation," Phys. Rev. Lett. 115, 241301 (2015) [arXiv:1506.04065 [hep-ph]].

[34] G. Ballesteros, J. Redondo, A. Ringwald and C. Tamarit, "Standard ModelaxionseesawHiggs portal inflation. Five problems of particle physics and cosmology solved in one stroke," JCAP 1708, no. 08, 001 (2017) [arXiv:1610.01639 [hep-ph]].

[35] A. Joti, A. Katsis, D. Loupas, A. Salvio, A. Strumia, N. Tetradis and A. Urbano, "(Higgs) vacuum decay during inflation," JHEP 1707, 058 (2017) [arXiv:1706.00792 [hep-ph]].

[36] J. R. Espinosa, D. Racco and A. Riotto, "Cosmological Signature of the Standard Model Higgs Vacuum Instability: Primordial Black Holes as Dark Matter," Phys. Rev. Lett. 120, no. 12, 121301 (2018) [arXiv:1710.11196 [hep-ph]].

[37] C. Han, S. Pi and M. Sasaki, "Quintessence Saves Higgs Instability," Phys. Lett. B 791, 314 (2019) [arXiv:1809.05507 [hep-ph]].

[38] A. Salvio, "Critical Higgs inflation in a Viable Motivated Model," Phys. Rev. D 99, no. 1, 015037 (2019) [arXiv:1810.00792 [hep-ph]].

[39] A. H. Guth and E. J. Weinberg, "Could the Universe Have Recovered from a Slow First Order Phase Transition?," Nucl. Phys. B 212, 321 (1983).

[40] G. F. Giudice and R. Rattazzi, "Living Dangerously with Low-Energy Supersymmetry," Nucl. Phys. B 757, 19 (2006) [hep-ph/0606105].

[41] S. Samuel, "The Standard model in its other phase," Nucl. Phys. B 597, 70 (2001) [hep-ph/9910559].

[42] N. Arkani-Hamed, S. Dimopoulos and S. Kachru, "Predictive landscapes and new physics at a TeV," hep-th/0501082.

[43] H. Friedrich, "On the existence of n-geodesically complete or future complete solutions of Einstein's field equations with smooth asymptotic structure," Commun. Math. Phys. 107, 587 (1986).

[44] P. Bizon and A. Rostworowski, "On weakly turbulent instability of anti-de Sitter space," Phys. Rev. Lett. 107, 031102 (2011) [arXiv:1104.3702 [gr-qc]].

[45] R. Bousso and J. Polchinski, "Quantization of four form fluxes and dynamical neutralization of the cosmological constant," JHEP 0006, 006 (2000) [hep-th/0004134].

[46] S. Kachru, R. Kallosh, A. D. Linde and S. P. Trivedi, "De Sitter vacua in string theory," Phys. Rev. D 68, 046005 (2003) [hep-th/0301240].

[47] L. Susskind, "The Anthropic landscape of string theory," In *Carr, Bernard (ed.): Universe or multiverse?* 247-266 [hep-th/0302219].

[48] M. R. Douglas, "The Statistics of string / M theory vacua," JHEP 0305, 046 (2003) [hep-th/0303194].

[49] A. Vilenkin, "The Birth of Inflationary Universes," Phys. Rev. D 27, 2848 (1983).

[50] A. D. Linde, "Eternal Chaotic Inflation," Mod. Phys. Lett. A 1, 81 (1986).

[51] A. D. Linde, "Eternally Existing Selfreproducing Chaotic Inflationary Universe," Phys. Lett. B 175, 395 (1986). 
[52] G. Obied, H. Ooguri, L. Spodyneiko and C. Vafa, "De Sitter Space and the Swampland," arXiv:1806.08362 [hep-th].

[53] P. Agrawal, G. Obied, P. J. Steinhardt and C. Vafa, "On the Cosmological Implications of the String Swampland," Phys. Lett. B 784, 271 (2018) [arXiv:1806.09718 [hep-th]].

[54] J. J. Heckman, C. Lawrie, L. Lin and G. Zoccarato, "F-theory and Dark Energy," arXiv:1811.01959 [hep-th].

[55] B. Freivogel, "Making predictions in the multiverse," Class. Quant. Grav. 28, 204007 (2011) [arXiv:1105.0244 [hep-th]].

[56] A. D. Linde and A. Mezhlumian, "Stationary universe," Phys. Lett. B 307, 25 (1993) [gr-qc/9304015].

[57] A. D. Linde, D. A. Linde and A. Mezhlumian, "From the Big Bang theory to the theory of a stationary universe," Phys. Rev. D 49, 1783 (1994) [gr-qc/9306035].

[58] J. Garcia-Bellido, A. D. Linde and D. A. Linde, "Fluctuations of the gravitational constant in the inflationary Brans-Dicke cosmology," Phys. Rev. D 50, 730 (1994) [astro-ph/9312039].

[59] A. Vilenkin, "Predictions from quantum cosmology," Phys. Rev. Lett. 74, 846 (1995) [gr-qc/9406010].

[60] A. De Simone, A. H. Guth, M. P. Salem and A. Vilenkin, "Predicting the cosmological constant with the scale-factor cutoff measure," Phys. Rev. D 78, 063520 (2008) [arXiv:0805.2173 [hep-th]].

[61] J. Garriga and A. Vilenkin, "Recycling universe," Phys. Rev. D 57, 2230 (1998) [astro-ph/9707292].

[62] J. Garriga, D. Schwartz-Perlov, A. Vilenkin and S. Winitzki, "Probabilities in the inflationary multiverse," JCAP 0601, 017 (2006) [hep-th/0509184].

[63] V. Vanchurin and A. Vilenkin, "Eternal observers and bubble abundances in the landscape," Phys. Rev. D 74, 043520 (2006) [hep-th/0605015].

[64] R. Bousso, "Holographic probabilities in eternal inflation," Phys. Rev. Lett. 97, 191302 (2006) [hepth/0605263].

[65] R. Bousso, "Complementarity in the Multiverse," Phys. Rev. D 79, 123524 (2009) [arXiv:0901.4806 [hep-th]].

[66] R. Bousso, B. Freivogel, S. Leichenauer and V. Rosenhaus, "A geometric solution to the coincidence problem, and the size of the landscape as the origin of hierarchy," Phys. Rev. Lett. 106, 101301 (2011) [arXiv:1011.0714 [hep-th]].

[67] R. Bousso, B. Freivogel and I. S. Yang, "Properties of the scale factor measure," Phys. Rev. D 79, 063513 (2009) [arXiv:0808.3770 [hep-th]].

[68] J. Garriga and A. Vilenkin, "Watchers of the multiverse," JCAP 1305, 037 (2013) [arXiv:1210.7540 [hep-th]].

[69] Y. Nomura, "Physical Theories, Eternal Inflation, and Quantum Universe," JHEP 1111, 063 (2011) [arXiv:1104.2324 [hep-th]].

[70] P. Anderson, H. J. Jensen, L. P. Oliveira and P. Sibani, "Evolution in complex systems," Complexity 10, 49 (2004) [cond-mat/0406689].

[71] J. C. Mauro and M. M. Smedskjaer, "Statistical mechanics of glass," Journal of Non-Crystalline Solids 396, 41 (2014). 
[72] F. Denef, "TASI lectures on complex structures," arXiv:1104.0254 [hep-th].

[73] F. Denef and M. R. Douglas, "Computational complexity of the landscape. I.," Annals Phys. 322, 1096 (2007) [hep-th/0602072].

[74] J. Halverson and F. Ruehle, "Computational Complexity of Vacua and Near-Vacua in Field and String Theory," Phys. Rev. D 99, no. 4, 046015 (2019) [arXiv:1809.08279 [hep-th]].

[75] F. Denef, M. R. Douglas, B. Greene and C. Zukowski, "Computational complexity of the landscape II — Cosmological considerations," Annals Phys. 392, 93 (2018) [arXiv:1706.06430 [hep-th]].

[76] R. Bousso, B. Freivogel, S. Leichenauer and V. Rosenhaus, "Eternal inflation predicts that time will end," Phys. Rev. D 83, 023525 (2011) [arXiv:1009.4698 [hep-th]].

[77] N. Bao, R. Bousso, S. Jordan and B. Lackey, "Fast optimization algorithms and the cosmological constant," Phys. Rev. D 96, no. 10, 103512 (2017) [arXiv:1706.08503 [hep-th]].

[78] S. R. Coleman, "The Fate of the False Vacuum. 1. Semiclassical Theory," Phys. Rev. D 15, 2929 (1977) Erratum: [Phys. Rev. D 16, 1248 (1977)].

[79] C. G. Callan, Jr. and S. R. Coleman, "The Fate of the False Vacuum. 2. First Quantum Corrections," Phys. Rev. D 16, 1762 (1977).

[80] S. R. Coleman and F. De Luccia, "Gravitational Effects on and of Vacuum Decay," Phys. Rev. D 21, 3305 (1980).

[81] J. Carifio, W. J. Cunningham, J. Halverson, D. Krioukov, C. Long and B. D. Nelson, "Vacuum Selection from Cosmology on Networks of String Geometries," Phys. Rev. Lett. 121, no. 10, 101602 (2018) [arXiv:1711.06685 [hep-th]].

[82] J. D. Noh and H. Rieger, "Random Walks on Complex Networks," Phys. Rev. Lett. 92, 118701 (2004).

[83] A. Barrat, M. Barthélemy and A. Vespignani, "Dynamical Processes on Complex Networks," Cambridge University Press, 361 p. (2012).

[84] N. Masuda, M. A. Porter and R. Lambiotte, "Random walks and diffusion on networks," Phys. Rept. 745, 1 (2018) [arXiv:1612.03281 [physics.soc-ph]].

[85] S. Redner, "A Guide to First-Passage Processes," Cambridge University Press, 328 p. (2001).

[86] C. P. Haynes and A. P. Roberts, "Global first-passage times of fractal lattices," Phys. Rev. E 78, 041111 (2008) [arXiv:0809.0563 [cond-mat.stat-mech]].

[87] Y. Lin and Z. Zhang, "Mean first-passage time for maximal-entropy random walks in complex networks," Nature, Scientific Reports, 4, 5365 (2014).

[88] V. Tejedor, O. Bénichou, and R. Voituriez, "Global mean first-passage times of random walks on complex networks," Phys. Rev. E 80, 065104(R) (2009) [arXiv:0909.0657 [cond-mat.stat-mech]].

[89] J. G. Kemeny and J. L. Snell, "Finite Markov Chains," Van Nostrand Comp. Int., New York, 1960.

[90] D. Schwartz-Perlov and A. Vilenkin, "Probabilities in the Bousso-Polchinski multiverse," JCAP 0606, 010 (2006) [hep-th/0601162].

[91] K. D. Olum and D. Schwartz-Perlov, "Anthropic prediction in a large toy landscape," JCAP 0710, 010 (2007) [arXiv:0705.2562 [hep-th]]. 
[92] K. M. Lee and E. J. Weinberg, "Decay of the True Vacuum in Curved Space-time," Phys. Rev. D 36, 1088 (1987).

[93] S. Havlin and D. Ben-Avraham, "Diffusion in disordered media," Advances in Physics 36, 695 (1987).

[94] A. Samarakoon et al., "Aging, memory, and nonhierarchical energy landscape of spin jam," Proc. Natl Acad. Sci USA 113, 11806 (2016) [arXiv:1707.03086 [cond-mat.dis-nn]].

[95] J. D. Bryngelson, J. N. Onuchic, N. D. Socci and P. G. Wolynes, "Funnels, Pathways and the Energy Landscape of Protein Folding: A Synthesis," Proteins-Struct. Func. and Genetics. 21, 167 (1995) [chem$\mathrm{ph} / 9411008]$.

[96] N. Gō, "Theoretical Studies of Protein Folding," Ann. Rev. Biophys. Bioeng. 12, 183 (1983)

[97] R. Unger and J. Moult, "Finding the Lowest Free-Energy Conformation of a Protein is an NP-hard Problem - Proof and Implications," Bull. Math. Bio. 55, 1183 (1993).

[98] C. Levinthal, "How to Fold Graciously," Mossbauer Spectroscopy in Biological Systems: Proceedings of a meeting held at Allerton House, Monticello, Illinois, Eds: J. T. P. DeBrunner and E. Munck, University of Illinois Press, pp. 22-24, (1969).

[99] A. Blum and R. L. Rivest, "Training a 3-node neural network is NP-complete," NIPS '88 Proceedings of the 1st International Conference on Neural Information Processing Systems, 494 (1988).

[100] H. Li, Z. Xu, G. Taylor, C. Studer and T. Goldstein, "Visualizing the Loss Landscape of Neural Nets," arXiv:1712.09913 [cs.LG].

[101] C. H. Martin and M. W. Mahoney, "Implicit Self-Regularization in Deep Neural Networks: Evidence from Random Matrix Theory and Implications for Learning," arXiv:1810.01075 [cs.LG].

[102] D. Sornette, "Critical phenomena in natural sciences: chaos, fractals, selforganization and disorder: concepts and tools," Springer-Verlag, Berlin, 2006.

[103] G. Pólya, "About a task of the probability calculation concerning the randomness in the road network," Math. Annals 84, 149 (1921).

[104] D. N. Page, "Information in black hole radiation," Phys. Rev. Lett. 71, 3743 (1993) [hep-th/9306083].

[105] U. H. Danielsson, D. Domert and M. E. Olsson, "Miracles and complementarity in de Sitter space," Phys. Rev. D 68, 083508 (2003) [hep-th/0210198].

[106] U. H. Danielsson and M. E. Olsson, "On thermalization in de Sitter space," JHEP 0403, 036 (2004) [hep-th/0309163].

[107] R. Z. Ferreira, M. Sandora and M. S. Sloth, "Asymptotic Symmetries in de Sitter and Inflationary Spacetimes," JCAP 1704, no. 04, 033 (2017) [arXiv:1609.06318 [hep-th]].

[108] R. Z. Ferreira, M. Sandora and M. S. Sloth, "Patient Observers and Non-perturbative Infrared Dynamics in Inflation," JCAP 1802, no. 02, 055 (2018) [arXiv:1703.10162 [hep-th]].

[109] P. Creminelli, S. Dubovsky, A. Nicolis, L. Senatore and M. Zaldarriaga, "The Phase Transition to Slow-roll Eternal Inflation," JHEP 0809, 036 (2008) [arXiv:0802.1067 [hep-th]].

[110] N. Arkani-Hamed, S. Dubovsky, A. Nicolis, E. Trincherini and G. Villadoro, "A Measure of de Sitter entropy and eternal inflation," JHEP 0705, 055 (2007) [arXiv:0704.1814 [hep-th]]. 
[111] R. Monasson et al., "Determining computational complexity from characteristic 'phase transitions'," Nature 400, 133 (1999).

[112] T. Hogg, B. A. Huberman and C. P. Williams, "Phase transitions and the search problem," Artificial Intelligence 81, 1 (1996).

[113] P. Bak, C. Tang and K. Wiesenfeld, "Self-organized criticality: An explanation of 1/f noise," Phys. Rev. Lett. 59381 (1987).

[114] P. Bak, "How Nature Works: The Science of Self-Organized Criticality," Springer-Verlag, New York (1996).

[115] H. J. Jensen, "Self-Organized Criticality," Cambridge University Press, Cambridge (1998).

[116] A. Roli, M. Villani, A. Filisetti and R. Serra, "Dynamical criticality: overview and open questions," J. of Systems Sci and Complexity, 31, 647 (2018).

[117] S. A. Kauffman, "The Origins of Order: Self-Organization and Selection in Evolution," Oxford University Press, Oxford (1993).

[118] E. Berlekamp, J. H. Conway and R. Guy, "Winning ways for your mathematical plays," Academic Press, New York, NY (1982).

[119] S. Wolfram, "Universality and complexity in cellular automata," Physica D 10, 1 (1984).

[120] N. H. Packard, "Adaptation toward the edge of chaos," Dynamic Patterns in Complex Systems 212, 293 (1988).

[121] C. G. Langton, "Computation at the edge of chaos: Phase transitions and emergent computation," Physica D 42, 12 (1990).

[122] J. P. Crutchfield and K. Young, "Inferring statistical complexity," Phys. Rev. Lett. 63, 105 (1989).

[123] J. P. Crutchfield and K. Young, "Computation at the onset of chaos," In W. H. Zurek, editor, Complexity, Entropy, and the Physics of Information, p. 223269, Addison-Wesley (1990).

[124] M. Mitchell, P. Hraber and J. P. Crutchfield, "Revisiting the Edge of Chaos: Evolving Cellular Automata to Perform Computations," Complex Systems 7, 89 (1993) [arXiv:adap-org/9303003].

[125] N. Bertschinger and T. Natschläger, "Real-time computation at the edge of chaos in recurrent neural networks," Neural Computation 16, 1413 (2004).

[126] J. Boedecker, O. Obst O, J. T. Lizier, N. M. Mayer and M. Asada, "Information processing in echo state networks at the edge of chaos," Theory in Biosciences 131, 205 (2012).

[127] M. Lukoševičius and H. Jaeger "Reservoir computing approaches to recurrent neural network training," Computer Science Review 3, 127 (2009).

[128] F. Matzner, "Neuroevolution on the Edge of Chaos," Proc. of the Genetic and Evolutionary Computation Conference, 465 (2017) [arXiv:1706.01330 [cs.NE]].

[129] M. Usher, M. Stemmler and Z. Olami, "Dynamic pattern formation leads to $1 / f$ noise in neural populations," Phys Rev Lett 74, 326 (1995).

[130] O. Kinouchi and M, Copelli, "Optimal Dynamical Range of Excitable Networks at Criticality," Nature Phys. 2, 348 (2006) [arXiv:q-bio/0601037 [q-bio.NC]].

[131] J. M. Beggs and D. Plenz, "Neuronal avalanches in neocortical circuits," J. Neurosci 23, 11167 (2003). 
[132] D. R. Chialvo, "Emergent complex neural dynamics," Nature Phys. 6, 744 (2010) [arXiv:1010.2530 [q-bio.NC]].

[133] T. Mora and W. Bialek, "Are biological systems poised at criticality?" J. Stat. Phys. 144, 268 (2011) [arXiv:1012.2242 [q-bio.QM]].

[134] M. Nykter et al., "Gene expression dynamics in the macrophage exhibit criticality," Proc. Natl Acad. Sci USA 105, 1897 (2008).

[135] C. Furusawa and K. Kaneko "Adaptation to optimal cell growth through self-organized criticality," Phys. Rev. Lett. 108, 208103 (2012).

[136] A. Cavagna et al., "Scale-free correlations in starling flocks," Proc. Natl Acad. Sci USA 107, 11865 (2010).

[137] W. Bialek et al., "Statistical mechanics for natural flocks of birds," Proc. Natl Acad. Sci USA 109, 4786 (2012) [arXiv:1107.0604 [physics.bio-ph]].

[138] G. H. Pyke, "Optimal Foraging Theory: A Critical Review," Ann. Rev. Ecol. Syst. 15, 523 (1984).

[139] J. Elias-Miro, J. R. Espinosa, G. F. Giudice, G. Isidori, A. Riotto and A. Strumia, "Higgs mass implications on the stability of the electroweak vacuum," Phys. Lett. B 709, 222 (2012) [arXiv:1112.3022 [hep-ph]].

[140] G. F. Giudice and A. Strumia, "Probing High-Scale and Split Supersymmetry with Higgs Mass Measurements," Nucl. Phys. B 858, 63 (2012) [arXiv:1108.6077 [hep-ph]].

[141] L. Smolin, "Did the Universe Evolve?," Class. Quant. Grav. 9, 173 (1992).

[142] L. Smolin, "The Fate of black hole singularities and the parameters of the standard models of particle physics and cosmology," gr-qc/9404011.

[143] L. Smolin, "Cosmology as a problem in critical phenomena," Lect. Notes Phys. 461, 184 (1996) [grqc/9505022].

[144] L. Smolin, "Using neutron stars and primordial black holes to test theories of quantum gravity," astro-ph/9712189.

[145] A. R. Brown, A. Dahlen and A. Masoumi, "Compactifying de Sitter space naturally selects a small cosmological constant," Phys. Rev. D 90, no. 12, 124048 (2014) [arXiv:1311.2586 [hep-th]].

[146] S. Weinberg, "A Priori probability distribution of the cosmological constant," Phys. Rev. D 61, 103505 (2000) [astro-ph/0002387].

[147] J. Garriga, A. Vilenkin and J. Zhang, "Non-singular bounce transitions in the multiverse," JCAP 1311, 055 (2013) [arXiv:1309.2847 [hep-th]].

[148] M. P. Salem, "Multiverse rate equation including bubble collisions," Phys. Rev. D 87, no. 6, 063501 (2013) [arXiv:1210.7181 [hep-th]].

[149] A. R. Brown and A. Dahlen, "Populating the Whole Landscape," Phys. Rev. Lett. 107, 171301 (2011) [arXiv:1108.0119 [hep-th]].

[150] Z. Zhang, T. Shan and G. Chen, "Random walks on weighted networks," Phys. Rev. E 87, 012112 (2013) [arXiv:1212.5998 [cond-mat.stat-mech]].

[151] J. Polchinski, "The Cosmological Constant and the String Landscape," hep-th/0603249. 
[152] T. M. Michelitsch et al.. "Recurrence of random walks with long-range steps generated by fractional Laplacian matrices on regular networks and simple cubic lattices," Journal of Phys. A: Math. and Theo. 50, 50, 505004 (2017) [arXiv:1707.05843 [cond-mat.stat-mech]].

[153] E. W. Montroll, "Random Walks on Lattices. III. Calculation of FirstPassage Times with Application to Exciton Trapping on Photosynthetic Units," J. Math. Phys. 10, 753 (1969).

[154] B. A. Huberman and T. Hogg, "Phase transitions in artificial intelligence systems," Artificial Intelligence 33, 155 (1987).

[155] I. P. Gent and T. Walsh, "The SAT Phase Transition," Proceedings of the 11th European Conference on Artificial Intelligence, A. Cohn (Ed.), John Wiley \& Sons, Ltd (1994).

[156] A. R. Brown and A. Dahlen, "Small Steps and Giant Leaps in the Landscape," Phys. Rev. D 82, 083519 (2010) [arXiv:1004.3994 [hep-th]].

[157] P. Burda, R. Gregory and I. Moss, "The fate of the Higgs vacuum," JHEP 1606, 025 (2016) [arXiv:1601.02152 [hep-th]].

[158] J. F. Gunion, H. E. Haber and M. Sher, "Charge / Color Breaking Minima and a-Parameter Bounds in Supersymmetric Models," Nucl. Phys. B 306, 1 (1988).

[159] J. A. Casas, A. Lleyda and C. Munoz, "Strong constraints on the parameter space of the MSSM from charge and color breaking minima," Nucl. Phys. B 471, 3 (1996) [hep-ph/9507294].

[160] A. Kusenko, P. Langacker and G. Segre, "Phase transitions and vacuum tunneling into charge and color breaking minima in the MSSM," Phys. Rev. D 54, 5824 (1996) [hep-ph/9602414].

[161] A. Strumia, "Charge and color breaking minima and constraints on the MSSM parameters," Nucl. Phys. B 482, 24 (1996) [hep-ph/9604417].

[162] S. Abel and T. Falk, "Charge and color breaking in the constrained MSSM," Phys. Lett. B 444, 427 (1998) [hep-ph/9810297].

[163] D. Chowdhury, R. M. Godbole, K. A. Mohan and S. K. Vempati, "Charge and Color Breaking Constraints in MSSM after the Higgs Discovery at LHC," JHEP 1402, 110 (2014) Erratum: [JHEP 1803, 149 (2018)] [arXiv:1310.1932 [hep-ph]].

[164] M. P. Hertzberg, "A Correlation Between the Higgs Mass and Dark Matter," Adv. High Energy Phys. 2017, 6295927 (2017) [arXiv:1210.3624 [hep-ph]].

[165] A. Linde and V. Vanchurin, "Towards a non-anthropic solution to the cosmological constant problem," arXiv:1011.0119 [hep-th].

[166] T. Banks, "Cosmological breaking of supersymmetry?," Int. J. Mod. Phys. A 16, 910 (2001) [hepth/0007146].

[167] Y. S. Piao, "Can the universe experience many cycles with different vacua?," Phys. Rev. D 70, 101302 (2004) [hep-th/0407258].

[168] Y. S. Piao, "Proliferation in Cycle," Phys. Lett. B 677, 1 (2009) [arXiv:0901.2644 [gr-qc]].

[169] M. C. Johnson and J. L. Lehners, "Cycles in the Multiverse," Phys. Rev. D 85, 103509 (2012) [arXiv:1112.3360 [hep-th]].

[170] J. L. Lehners, "Eternal Inflation With Non-Inflationary Pocket Universes," Phys. Rev. D 86, 043518 (2012) [arXiv:1206.1081 [hep-th]]. 
[171] G. M. Viswanathan et al., "Optimizing the success of random searches," Nature 381, 413 (1996).

[172] M. A. Lomholt, K. Tal, R. Metzler and K. Joseph, "Lévy strategies in intermittent search processes are advantageous," Proc. Natl. Acad. Sci. 105, 11055 (2008).

[173] O. Bénichou, C. Loverdo, M. Moreau and R. Voituriez, "Intermittent search strategies," Rev. Mod. Phys. 83, 81 (2011).

[174] O. Bénichou, M. Coppey, M. Moreau, P.-H. Suet and R. Voituriez, "Optimal search strategies for hidden targets," Phys. Rev. Lett. 94, 198101 (2005).

[175] F. Di Patti, D. Fanelli and F. Piazza, "Optimal search strategies on complex multi-linked networks," Nature, Scientific Reports, 5, 9869 (2015) [arXiv:1408.1603 [physics.soc-ph]].

[176] W. J. O'Brien, B. I. Evans and H. I. Browman, "Flexible search tactics and efficient foraging in saltatory searching animals," Oecologia 80, 100 (1989).

[177] G. M. Viswanathan et al., "Lévy flight search patterns of wandering albatrosses," Nature 401, 911 (1999).

[178] D. W. Sims et al., "Scaling laws of marine predator search behaviour," Nature 451, 1098 (2008).

[179] N. E. Humphries et al., "Environmental context explains Lévy and Brownian movement patterns of marine predators," Nature 465, 1066 (2010).

[180] D. A. Raichlen et al., "Evidence of Lvy walk foraging patterns in human huntergatherers," Proc. Natl. Acad. Sci. 131, 18616 (2013).

[181] S. Brin and L. Page, "The anatomy of a large-scale hypertextual Web search engine," Computer Networks and ISDN Systems 30, 107 (1998).

[182] D. F. Gleich, "PageRank Beyond the Web," Soc. for Ind. and Appl. Math. Rev. 57, 321 (2015) [arXiv:1407.5107 [cs.SI]].

[183] T. Banks, M. Dine and E. Gorbatov, "Is there a string theory landscape?," JHEP 0408, 058 (2004) [hep-th/0309170].

[184] F. Gmeiner, R. Blumenhagen, G. Honecker, D. Lust and T. Weigand, "One in a billion: MSSM-like D-brane statistics," JHEP 0601, 004 (2006) [hep-th/0510170].

[185] M. Tegmark, "On the dimensionality of space-time," Class. Quant. Grav. 14, L69 (1997) [grqc/9702052].

[186] G. Grinstein, D.-H. Lee, and S. Sachdev, "Conservation laws, anisotropy, and "self-organized criticality" in noisy nonequilibrium systems," Phys. Rev. Lett. 64, 1927 (1990).

[187] G. Grinstein, "Generic scale invariance in classical nonequilibrium systems (invited)," Journal of Applied Physics 69, 5441 (1991). 